Pure and Applied Mathematics Quarterly

Volume 4, Number 3

(Special Issue: In honor of

Fedor Bogomolov, Part 2 of 2$)$

$651-714,2008$

\title{
Coherent Sheaves on General K3 Surfaces and Tori
}

\author{
Misha Verbitsky ${ }^{1}$
}

Abstract: Let $M$ be a K3 surface or an even-dimensional compact torus. We show that the category of coherent sheaves on $M$ is independent of the choice of the complex structure, if this complex structure is generic.

\section{Contents}

1. Introduction 653

1.1. An overview 653

1.2. A plan of the proof 654

1.3. Flat bundles on complex tori 656

1.4. Reflexive sheaves on hyperkähler manifolds 658

1.5. Contents 659

2. Hyperkähler manifolds 661

2.1. Hyperkähler manifolds and quaternionic action 661

2.2. Generic induced complex structures and trianalytic subvarieties 662

3. Hyperholomorphic bundles 664

3.1. Hyperholomorphic connections 664

3.2. Hyperholomorphic bundles, Yang-Mills connections and stability 665

Received Febuary 27, 2006.

${ }^{1}$ The author is partially supported by CRDF grant RM1-2354-MO-02. 
4. Generic complex structures

on hyperkähler manifolds

667

5. Category of reflexive sheaves 669

6. Twistor formalism and cohomology 670

6.1. Twistor spaces 670

6.2. Twistor formalism for vector bundles 670

6.3. Cohomology of $\operatorname{Tw}(B) \quad 672$

7. Local geometry of the twistor space 675

7.1. Twistor lines and local geometry 675

7.2. Local trivialization of the sheaf of differential operators 678

$\begin{array}{lll}\text { 7.3. Twistor space and cohomology with support } & 682\end{array}$

8. Coherent sheaves on generic compact tori 686

8.1. Reflexive sheaves on generic complex tori are bundles 686

9. Yoneda extension in abelian categories 694

9.1. Abelian categories of cohomological dimension $\leqslant 1 . \quad 694$

9.2. Functors on abelian categories of finite length 695

10. Reflexive sheaves on twistor spaces 697

10.1. Category $\mathcal{C}_{I}^{t w}:$ the definition 698

10.2. Reflexive sheaves on generic compact torus and the category $\mathcal{C}_{I}^{t w} \quad 702$

10.3. Reflexive sheaves on hyperkähler surfaces 706

11. Coherent sheaves with isolated singularities 708

$\begin{array}{ll}\text { References } & 713\end{array}$ 


\section{INTRODUCTION}

1.1. An overview. A hyperkähler manifold is a Riemannian manifold $M$ equipped with an orthogonal action of quaternion algebra in its tangent space $T M$, in such a way that for all $L \in \mathbb{H}, L^{2}=-1$, the $L$ induces a Kähler structure on $M$.

Let $M$ be a hyperkähler manifold, and $I \in \mathbb{H}$ a quaternion such that $I^{2}=$ -1 . By $(M, I)$ we understand the $M$ considered as a Kähler manifold, with the complex structure $I$.

We study the category of coherent sheaves on $(M, I)$. This question can be stated more algebraically as follows. Using the linear algebra of the quaternionic action, it is easy to construct a holomorphic symplectic form on $(M, I)$, that is, a holomorphic, closed, nowhere degenerate $(2,0)$-form. Conversely, every compact holomorphic symplectic Kähler manifold admits, by Calabi-Yau theorem, a unique hyperkähler metric in a given Kähler class. Details of this correspondence can be found e.g. in [Bes].

The subject of this paper can be understood algebraically as the study of coherent sheaves on compact holomorphically symplectic Kähler manifolds.

A holomorphically symplectic manifold has trivial canonical class; the canonical class is trivialized by taking the top exterior power of the holomorphic symplectic form. Mirror conjecture, in the form proposed by M. Kontsevich ([Kon]), relates the category of coherent sheaves on a Calabi-Yau manifold and the Gromov-Witten geometry of the Lagrangian cycles on its mirror partner. More precisely, this version of the mirror conjecture states that the derived category of coherent sheaves should be equivalent to the Fukaya category of the mirror dual manifold.

The mirror partner of a holomorphically symplectic manifold is again holomorphically symplectic. However, a generic deformation of a holomorphically symplectic manifold does not admit holomorphic curves, so one would expect that all Gromov-Witten-type invariants (including the Fukaya category) are (in some sense) trivial.

In this paper we prove the mirror counterpart of this conjecture, for $M$ a K3 surface or a compact complex torus. 
Theorem 1.1: Let $M_{1}, M_{2}$ be K3 surfaces or compact complex tori of dimension $2 d$. Assume that $M_{1}, M_{2}$ are generic in the sense of having no non-trivial integer $(p, p)$-cycles, for $0<p<2 d$, and $\mathcal{C}_{1} \mathcal{C}_{2}$ be the category of coherent sheaves on $M_{1}, M_{2}$. Then $\mathcal{C}_{1}$ is (non-canonically) equivalent to $\mathcal{C}_{2}$.

Proof: See Theorem 11.6.

In the algebraic situation, D. Orlov $([\mathrm{O}])$ studied how the derived category of coherent sheaves behaves when one changes the complex structure of a K3 surface. This is not the situation we work in, because generic complex structures on hyperkähler manifolds are never algebraic.

The derived category of coherent sheaves is a weaker invariant than the category of coherent sheaves itself. Using the Fourier-Mukai transform, D. Orlov shows that the derived categories of coherent sheaves on the projective K3 surfaces $M$ and $M^{\prime}$ are equivalent if and only if the Hodge lattices of trancendental cycles in $H^{2}(M), H^{2}\left(M^{\prime}\right)$ are isometric.

1.2. A plan of the proof. The proof of Theorem 1.1 is based on hyperkähler geometry and on the theory of reflexive sheaves. A coherent sheaf is called reflexive if it is isomorphic to its second dual (Definition 5.1).

Let $M$ be a compact hyperkähler manifold, and $S$ the 2-sphere of complex structures induced by the quaternionic action. There is a natural $S U(2)$-action on the cohomology $H^{*}(M)$ of $M$ (Lemma 2.3). A complex structure $L \in S$ is called generic (Definition 2.8) if all $(p, p)$-cycles on $(M, L)$ are $S U(2)$-invariant.

In [V1] it was proven that all induced complex structures $L \in S$ outside of a countable set are generic (Proposition 2.9).

If $L$ is generic, then all integer $(1,1)$-classes are $S U(2)$-invariant, hence are of degree zero (Lemma 3.8). Therefore, all vector bundles on $(M, L)$ are semistable of degree zero. ${ }^{1}$

\footnotetext{
${ }^{1}$ Throughout this paper, stability, polystability and semistability of vector bundles and coherent sheaves is understood in the sense of Mumford-Takemoto, see Definition 3.3. The degree of a coherent sheaf $F$ on a Kähler manifold $M$ is equal to $\int_{M} c_{1}(F) \wedge \omega^{n-1}$, where $n=\operatorname{dim}_{\mathbb{C}} M$ and $\omega$ is the Kähler form.
} 
Theorem 1.2: Let $M$ be a hyperkähler K3 surface or a compact hyperkähler torus of real dimension $2 d$. Let $L_{1}, L_{2}$ induced complex structures, and $\mathcal{C}_{1}^{r}, \mathcal{C}_{2}^{r}$ the categories of reflexive sheaves on $\left(M, L_{1}\right)$ and $\left(M, L_{2}\right)$ (Definition 5.6). Assume that $\left(M, L_{1}\right)$ and $\left(M, L_{2}\right)$ have no non-trivial integer $(p, p)$-cycles, for $0<p<d{ }^{2}$ Then the categories $\mathcal{C}_{1}^{r}, \mathcal{C}_{2}^{r}$ are equivalent.

Proof: This is Theorem 10.1.

The proof of Theorem 1.2 is based on the following idea, which is due to [KV]. Let $L$ be a generic induced complex structure on $M$. Every bundle on $(M, L)$ is semistable. In $[\mathrm{KV}]$ we studied the twistor correspondence, which allows one to construct a Yang-Mills bundle $\operatorname{Tw}(B)$ on the twistor space from a stable bundle $B$ on $(M, L)$ (see Theorem 6.2). This correspondence is actually injective: restricting $\operatorname{Tw}(B)$ to $(M, L) \subset \operatorname{Tw}(M)$, one obtains $B$ again. In $[\mathrm{KV}]$ we outline the set of conditions which guarantees that a given holomorphic bundle on the twistor space is obtained from a bundle with connection on $(M, L)$ (see Theorem 6.2). This formalism is functorial; we call it the direct and inverse twistor transform.

In this paper we apply the twistor formalism to the semistable bundles. Given a semistable bundle on $(M, L)$, we lift it to a holomorphic bundle on a twistor space, thus obtaining an equivalence between the category of semistable bundles on $(M, L)$ and a certain subcategory of holomorphic bundles on the twistor space.

If we replace the category $\mathcal{C}^{b}$ of holomorphic vector bundles by its subcategory $\mathcal{C}^{s t}$ of polystable bundles ${ }^{3}$, then Theorem 1.2 is well known (see [V4]). The equivalence $\mathcal{C}_{1}^{s t} \cong \mathcal{C}_{2}^{s t}$ is canonical.

\footnotetext{
${ }^{2}$ This is equivalent to $\left(M, L_{i}\right)$ being Mumford-Tate generic; see Section 4 for details.

${ }^{3}$ A polystable bundle (Definition 3.3 ) is by definition a direct sum of stable bundles of the same slope.
} 
The sphere $S^{2}$ of induced complex structures gives a holomorphic embedding $\mathbb{C} P^{1} \stackrel{j}{\hookrightarrow} \mathcal{M}$, where $\mathcal{M}$ is the moduli of complex structures on $M$. Let $I_{1}, I_{2} \in j\left(\mathbb{C} P^{1}\right)$ be generic induced complex structures. By Theorem 1.2, the corresponding categories of holomorphic vector bundles are isomorphic. In [V2] it was proven that any pair of complex Kähler structures $I_{1}, I_{2} \in \mathcal{M}$ within the same component of the moduli space can be connected by a sequence of rational curves of form $\mathbb{C} P^{1} \stackrel{j_{k}}{\hookrightarrow} \mathcal{M}$ associated with a sequence of hyperkähler structures. Moreover, these hyperkähler structures can be chosen in such a way that the sequential intersection points $j_{k}\left(\mathbb{C} P^{1}\right) \cap j_{k+1}\left(\mathbb{C} P^{1}\right)$ correspond to generic complex structures (Theorem 4.5).

Therefore, Theorem 1.2 shows that the category of reflexive sheaves on $(M, I)$ is independent from the complex structure $I$, as long as $I$ remains generic.

1.3. Flat bundles on complex tori. Let $T$ be a generic compact hyperkähler torus of complex dimension $>2$, and $I$ a generic induced complex structure. Then, all coherent sheaves on $(T, I)$ have isolated singularities and locally free reflexizations (Theorem 8.1).

Denote by $\mathcal{C}^{b}(T, I)$ the category of holomorphic vector bundles on $(T, I)$. To prove that $\mathcal{C}^{b}(T, I)$ is independent from the choice of generic induced complex structure $I$, we produce a (non-Hermitian) flat connection $\nabla_{B}$ on a given bundle $B$ on $(T, I)$, in a functorial way. For $B$ stable, $\nabla$ coincides with the Yang-Mills connection produced by the Uhlenbeck-Yau theorem (Theorem 3.5).

Let $I^{\prime}$ be an arbitrary induced complex structure. Taking the $(0,1)$-part of $\nabla_{B}$ with respect to $I^{\prime}$, we obtain a holomorphic bundle $B_{I^{\prime}}$ over $\left(T, I^{\prime}\right)$. We show that this gives an equivalence between the respective categories of holomorphic vector bundles $\mathcal{C}^{b}(M, I)$ and $\mathcal{C}^{b}\left(M, I^{\prime}\right)$, if $I^{\prime}$ is also generic.

The singularities are dealt with in the same fashion. For any $x \in M$, we identify the neighbourhood $U_{I}, U_{I^{\prime}}$ of $x$ in $(M, I)$ and $\left(M, I^{\prime}\right)$ (Subsection 7.1). We show that this identification is compatible with the connection $\nabla_{B}$, in such a way that the restriction of $B$ to $U_{I}$ is isomorphic to the restriction of $B_{I^{\prime}}$ to $U_{I^{\prime}}$ (Subsection 7.1). This allows to "glue in" the isolated singularities to $B$ and $B_{I^{\prime}}$ in a way which is canonical and compatible with $\nabla_{B}$. 
The twistor correspondence (Theorem 6.2) gives a way to speak of connections on $B$ algebraically: we identify the connections with $S U(2)$-invariant curvature and the lifts of the holomorphic structure from $B$ to the twistor space. Throughout this paper, we speak of holomorphic structures on the bundles in the twistor space, but Theorem 6.2 claims that this is the same as to speak of connections.

If we work over a torus, flatness is a more natural condition than $S U(2)$ invariance of the curvature. We propose a way to prove Theorem 1.2 on a torus without using the hyperkähler structure, as follows.

Let $(B, \nabla)$ be a flat bundle on a complex manifold. Taking the $(0,1)$-part of $\nabla$, we obtain a holomorphic structure operator on $B$. This gives a functor $\tau$ from the category of flat bundles to the category of holomorphic bundles. In this paper, we construct the "inverse" functor, mapping holomorphic bundles to flat ones.

Claim 1.3: Let $T$ be a compact $d$-dimensional complex torus, without nontrivial integer $(p, p)$-cycles in cohomology, $0<p<d$, and $\mathcal{C}^{b}(T)$ the category of holomorphic vector bundles on $T$. Assume that $d>2$. Then there exists a functor $B \longrightarrow\left(B, \nabla_{B}\right)$ from $\mathcal{C}^{b}(T)$ to the category of flat bundles on $T$, such that for any $B$, the holomorphic bundle $\tau(B, \nabla)$ is isomorphic to $B$, and for $B$ stable, the connection $\nabla$ is Hermitian.

Proof: This is Corollary 8.7.

It seems that it is possible to deduce the equivalence of the categories of vector bundles from Claim 1.3. We take a vector bundle $B$ and obtain the flat connection $\nabla$ on $B$ as in Claim 1.3. We change the complex structure $I$ on $T$ to a sufficiently close $I^{\prime}$ and restrict $(B, \nabla)$ to $\left(T, I^{\prime}\right)$, obtaining a holomorphic bundle $B_{I^{\prime}}$ on $\left(T, I^{\prime}\right)$. Since the stable bundles correspond to flat Hermitian connections, this construction will identify the stable bundles on $(T, I)$ and $\left(T, I^{\prime}\right)$. To prove that we obtained an equivalence, it remains to show that this functor preserves the 
Ext ${ }^{1}$-groups (see Corollary 9.7). However, the Ext classes can be represented by parallel forms on $(T, I),\left(T, I^{\prime}\right)$, and as we change the complex structure from $I$ to $I^{\prime}$, parallel forms remain parallel, and the corresponding cohomology classes are non-zero, if $I^{\prime}$ is sufficiently close to $I$.

To prove the equivalence of the respective categories of coherent sheaves, we use Theorem 8.1, stating that on a generic compact complex torus, all coherent sheaves have isolated singularities and smooth reflexive hulls. To pass from a bundle to a coherent sheaf, we need to "glue in" isolated singularities to a holomorphic vector bundle $B$.

Using the flat structure obtained in Claim 1.3, we can introduce flat coordinates on the tori $(T, I),\left(T, I^{\prime}\right)$ in a neighbourhood of a given point $x$, in such a way that the flat structure on $B$ will be compatible with these coordinates. This gives a natural functorial isomorphism between $B$ and $B_{I^{\prime}}$ in a neighbourhood of $x$. As in the proof of Theorem 11.5, we could "glue in" the singularities to $B$ and $B_{I^{\prime}}$ simultaneously, obtaining the isomorphism of the categories of coherent sheaves.

1.4. Reflexive sheaves on hyperkähler manifolds. The main motivation of Theorem 1.2 is the following. Suppose that $X$ is a complex manifold without divisors. We identify the category of reflexive sheaves $\mathcal{C}^{r}(X)$ with the category

$$
\underset{\lim }{\longrightarrow} \mathcal{C}^{b}(X \backslash S),
$$

where the limit is taken over all increasing sequences of closed complex analytic subvarieties $S \subset X, \operatorname{codim}_{X} S \geqslant 2$, and $\mathcal{C}^{b}(X \backslash S)$ is the category of holomorphic vector bundles on $X \backslash S$ which can be extended to $X$ as coherent sheaves (Definition 5.6). From this description it is clear that every object of $\mathcal{C}^{r}(X)$ is a finite extension of a sequence of simple objects. We say that an abelian category with this property has finite length, or satisfies the ascending and the descending chain conditions.

Now consider a compact hyperkähler manifold $M$ and let $I$ be a generic complex structure induced by quaternions. All complex subvarieties of $(M, I)$ are hyperkähler, hence have even codimension ([V1]). Therefore, $(M, I)$ has no divisors. The set of simple objects of $\mathcal{C}^{r}(M, I)$ is independent from $I$ as long as $I$ stays generic ([V4]). The Ext* groups between simple objects can also be computed (see Proposition 6.3), and (at least for locally free sheaves) these groups are independent from $I$ as well. We arrive at the following situation, which was 
known in many instances since 1990-ies. Given a hyperkähler manifold $M$, and generic complex structures $I_{1}, I_{2}$ in the same connected component of the moduli space, consider the categories of reflexive sheaves $\mathcal{C}^{r}\left(M, I_{1}\right)$ and $\mathcal{C}^{r}\left(M, I_{2}\right)$. Then

(i): These categories are of finite length, that is, satisfy the ascending and the descending chain conditions.

(ii): There is a natural equivalence $R$ between the simple objects of $\mathcal{C}^{r}\left(M, I_{1}\right)$ and $\mathcal{C}^{r}\left(M, I_{2}\right)$ (see [V4]).

(iii): If $M$ is a K3 surface or a torus, then the differential graded algebra $\operatorname{Ext}^{*}(B, B)$ is isomorphic to $\operatorname{Ext}^{*}(R(B), R(B))$, for any simple object $B$ in $\mathcal{C}^{r}\left(M, I_{1}\right)$.

The statement (iii) is known only for vector bundles ([V0]). Fortunately, reflexive sheaves on $M$ are locally free, if $M$ is a generic torus or a K3 surface (see Theorem 8.1).

It seems that the conditions (i)-(iii) are already sufficient to prove the equivalence of the categories $\mathcal{C}^{r}\left(M, I_{1}\right)$ and $\mathcal{C}^{r}\left(M, I_{2}\right)$, so one could prove Theorem 1.2 in an algebraic fashion via the theory of abelian categories. In the present paper we work in a different direction. We construct a subcategory $\mathcal{C}_{I}^{t w}$ of the category $\mathcal{C}^{r}(\operatorname{Tw}(M))$ of reflexive sheaves on the twistor space of $M$ (see Subsection 10.1). We show that the natural restriction functor from $\operatorname{Tw}(M)$ to $\left(M, I_{i}\right)$ induces an isomorphism between $\mathcal{C}_{I}^{t w}$ and $\mathcal{C}^{r}\left(M, I_{i}\right)$. This implies that the categories $\mathcal{C}^{r}\left(M, I_{1}\right)$ and $\mathcal{C}^{r}\left(M, I_{2}\right)$ are equivalent. Varying the hyperkähler structure, we obtain the same result for all generic complex structures.

1.5. Contents. This paper is organized as follows.

- This Introduction explains some ideas used in this paper, in a heuristic fashion.

- In Sections 2 - 3 we recall some results and definitions from the literature. We relate some basic facts from hyperkähler geometry, Yang-Mills theory and geometry of stable bundles.

- In Section 4, we study the moduli of complex structures on hyperkähler manifolds. We define the Mumford-Tate generic complex structures and show that these can be connected by a sequence of hyperkähler structures.

- In Section 5 we recall some standard results from the theory of reflexive sheaves (see e.g. $[\mathrm{OSS}]$ ). 
- In Section 6 we deal with the twistor formalizm introduced in [KV]. Let $M$ be a compact hyperkähler manifold, and $I$ a generic induced complex structure. Given a stable bundle $B$ on $(M, I)$, we construct a holomorphic vector bundle $\operatorname{Tw}(B)$ on its twistor space. We compute the cohomology of $\operatorname{Tw}(B)$ it terms of $H^{*}(B)$.

- In Section 7 we study the local geometry of the twistor space $\operatorname{Tw}(M)$, using the rational curves on $\operatorname{Tw}(M)$. Given a bundle $F$ on $\operatorname{Tw}(M)$ obtained from twistor transform, we trivialize $F$ in a neighbourhood of $\left(\mathbb{C} P^{1} \backslash I\right) \subset \operatorname{Tw}(M)$, where $\mathbb{C} P^{1}=s_{x}$ is the curve of form

$$
\mathbb{C} P^{1} \times\{x\} \subset \mathbb{C} P^{1} \times M=\operatorname{Tw}(M)
$$

and $I \subset s_{x}$ is an arbitrary point. We study the associated trivializations on the sheaves of differential operators and local cohomology. In particular, we prove that a differential operator or a section of the local cohomology sheaf in a neighbourhood of $s_{x} \backslash I$ can be extended to the whole $s_{x}$ if it is compatible with the trivialization.

- In Section 8 we study the coherent sheaves on a generic compact complex torus $T, \operatorname{dim}_{\mathbb{C}} \geqslant 3$. We show that all reflexive sheaves on $T$ are locally free. We construct a natural flat connection on any holomorphic bundle on $T$.

- In Section 9 we deal with formal properties of abelian categories satisfying the ascending and descending chain conditions (such categories are also called of finite length). We define the abelian categories of cohomological dimension $\leqslant 1$ and give a set of criteria which imply that a given category has cohomological dimension $\leqslant 1$. Given a functor of categories of finite length and cohomological dimension $\leqslant 1$, we show that it is an equivalence of categories if it is an equivalence on the simple objects and on Ext ${ }^{1}$-groups.

- In Section 10 we show that the categories of reflexive sheaves on $(M, I)$ and $\left(M, I^{\prime}\right)$ are equivalent if $M$ is a generic K3 surface or a hyperkähler torus, and $I, I^{\prime}$ are generic induced complex structures. For a surface, we use the results about local cohomology obtained earlier. For $M$ a torus of $\operatorname{dim}_{\mathbb{C}}>2$, we use the flat connection constructed in Section 8 on every holomorphic bundle.

- In Section 11, we apply the local geometry of the twistor space to "glue in" the singularities to vector bundles in a canonical way. This is used to 
show that the category of coherent sheaves with isolated singularities and smooth reflexizations on $(M, I)$ is independent from the choice of induced complex structure $I$, if $I$ remains generic.

\section{HYPERKÄHLER MANIFOLDS}

In this section we reproduce well known results from hyperkähler geometry, for later use. We follow [Bes] and [V1].

2.1. Hyperkähler manifolds and quaternionic action. Definition 2.1: ([Bes]) A hyperkähler manifold is a Riemannian manifold $M$ endowed with three complex structures $I, J$ and $K$, such that the following holds.

(i): the metric on $M$ is Kähler with respect to these complex structures and (ii): $I, J$ and $K$, considered as endomorphisms of a real tangent bundle, satisfy the relation $I \circ J=-J \circ I=K$.

The notion of a hyperkähler manifold was introduced by E. Calabi ([C]).

Clearly, a hyperkähler manifold has a natural action of the quaternion algebra $\mathbb{H}$ in its real tangent bundle $T M$. Therefore its complex dimension is even. For each quaternion $L \in \mathbb{H}, L^{2}=-1$, the corresponding automorphism of $T M$ is an almost complex structure. It is easy to check that this almost complex structure is integrable $([\mathrm{Bes}])$.

Definition 2.2: Let $M$ be a hyperkähler manifold, and $L$ a quaternion satisfying $L^{2}=-1$. The corresponding complex structure on $M$ is called an induced complex structure. The $M$, considered as a Kähler manifold, is denoted by $(M, L)$. In this case, the hyperkähler structure is called compatible with the complex structure $L$.

Let $M$ be a hyperkähler manifold. We identify the group $S U(2)$ with the group of unitary quaternions. This gives a canonical action of $S U(2)$ on the tangent 
bundle, and all its tensor powers. In particular, we obtain a natural action of $S U(2)$ on the bundle of differential forms.

The following lemma is clear.

Lemma 2.3: The action of $S U(2)$ on differential forms commutes with the Laplacian.

Proof: This is Proposition 1.1 of [V1].

Thus, for compact $M$, we may speak of the natural action of $S U(2)$ in cohomology.

Further in this article, we use the following statement.

Lemma 2.4: Let $\eta$ be a differential form over a hyperkähler manifold $M$. The form $\eta$ is $S U(2)$-invariant if and only if it is of Hodge type $(p, p)$ with respect to all induced complex structures on $M$.

Proof: Let $I$ be an induced complex structure, and $\mathfrak{u}_{I}: U(1) \longrightarrow S U(2)$ the corresponding embedding, induced by the map $\mathbb{R}=\mathfrak{u}(1) \longrightarrow \mathfrak{s u}(2), 1 \longrightarrow I$. The Hodge decomposition on $\Lambda^{*}(M)$ coincides with the weight decomposition of the $U(1)$-action $\mathfrak{u}_{I}$. An $S U(2)$-invariant form is also invariant with respect to $\mathfrak{u}_{I}$, and therefore has Hodge type $(p, p)$. Conversely, if a $\eta$ is invariant with respect to $\mathfrak{u}_{I}$, for all induced complex structures $I$, then $\eta$ is invariant with respect to the Lie group $G$ generated by these $U(1)$-subgroups of $S U(2)$. A trivial linear-algebraic argument ensures that the group $G$ coincides with the whole $S U(2)$. This proves Lemma 2.4.

2.2. Generic induced complex structures and trianalytic subvarieties. Let $M$ be a compact hyperkähler manifold, $\operatorname{dim}_{\mathbb{R}} M=2 m$. 
Definition 2.5: Let $N \subset M$ be a closed subset of $M$. Then $N$ is called trianalytic if $N$ is a complex analytic subset of $(M, L)$ for any induced complex structure $L$.

Let $I$ be an induced complex structure on $M$, and $N \subset(M, I)$ be a closed analytic subvariety of $(M, I), \operatorname{dim}_{\mathbb{C}} N=n$. Consider the homology class represented by $N$. Let $[N] \in H^{2 m-2 n}(M)$ denote the Poincare dual cohomology class, so-called fundamental class of $N$. Recall that the hyperkähler structure induces the action of the group $S U(2)$ on the space $H^{2 m-2 n}(M)$.

Theorem 2.6: Assume that $[N] \in H^{2 m-2 n}(M)$ is invariant with respect to the action of $S U(2)$ on $H^{2 m-2 n}(M)$. Then $N$ is trianalytic.

Proof: This is Theorem 4.1 of [V1].

Remark 2.7: Trianalytic subvarieties have an action of the quaternion algebra in the tangent bundle. In particular, the real dimension of such subvarieties is divisible by 4 .

Definition 2.8: Let $M$ be a compact hyperkähler manifold, and $I$ an induced complex structure. We say that $I$ is of general type or generic with respect to the hyperkähler structure on $M$, if all elements of the group

$$
\bigoplus_{p} H^{p, p}(M) \cap H^{2 p}(M, \mathbb{Z}) \subset H^{*}(M)
$$

are $S U(2)$-invariant.

Proposition 2.9: Let $M$ be a compact hyperkähler manifold, and $S$ the set of induced complex structures over $M$. Denote by $S_{0} \subset S$ the set of generic induced complex structures. Then $S_{0}$ is dense in $S$. Moreover, the complement $S \backslash S_{0}$ is countable.

Proof: This is Proposition 2.2 from [V1] 
Theorem 2.6 has the following immediate corollary:

Corollary 2.10: Let $M$ be a compact hyperkähler manifold, and $I$ a generic induced complex structure. Let $X \subset(M, I)$ be a closed complex analytic subvariety. Then $X$ is trianalytic. In particular, $X$ has even complex dimension

Throughout this paper, except Section 8, a weaker form of "generic" can be used: we could say that $I$ is generic if all integer $(p, p)$-cycles are $S U(2)$-invariant, for $p=1,2$.

\section{HyPERHOLOMORPHIC BUNDLES}

3.1. Hyperholomorphic connections. This Subsection contains several versions of a definition of hyperholomorphic connection in a complex vector bundle over a hyperkähler manifold. We follow [V0].

Let $B$ be a holomorphic vector bundle over a complex manifold $X, \nabla$ a connection in $B$ and $\Theta \in \Lambda^{2} \otimes \operatorname{End}(B)$ be its curvature. This connection is called compatible with the holomorphic structure if $\nabla_{\gamma}(\zeta)=0$ for any holomorphic section $\zeta$ and any antiholomorphic tangent vector field $\gamma \in T^{0,1}(X)$. If there exists a holomorphic structure compatible with the given Hermitian connection then this connection is called the Chern connection, or integrable.

One can define the Hodge decomposition in the space of differential forms with coefficients in any complex bundle, in particular, $\operatorname{End}(B)$.

Theorem 3.1: Let $\nabla$ be a Hermitian connection in a complex vector bundle $B$ over a complex manifold $X$. Then $\nabla$ is a Chern connection if and only if $\Theta \in \Lambda^{1,1}(X, \operatorname{End}(B))$, where $\Lambda^{1,1}(X, \operatorname{End}(B))$ denotes the forms of Hodge type $(1,1)$. Also, the holomorphic structure compatible with $\nabla$ is unique.

Proof: This is Proposition 4.17 of [Kob], Chapter I. 
This proposition is a version of Newlander-Nirenberg theorem. For vector bundles, it was proven by M. Atiyah and R. Bott.

Definition 3.2: Let $B$ be a Hermitian vector bundle with a connection $\nabla$ over a hyperkähler manifold $M$. Then $\nabla$ is called hyperholomorphic if $\nabla$ is a Chern connection with respect to each of the complex structures induced by the hyperkähler structure.

As follows from Theorem 3.1, $\nabla$ is hyperholomorphic if and only if its curvature $\Theta$ is of Hodge type $(1,1)$ with respect to any of the complex structures induced by a hyperkähler structure.

As follows from Lemma 2.4, $\nabla$ is hyperholomorphic if and only if $\Theta$ is an $S U(2)$-invariant differential form.

\subsection{Hyperholomorphic bundles, Yang-Mills connections and stability.} Definition 3.3: Let $F$ be a coherent sheaf over an $n$-dimensional compact Kähler manifold $M$. We define the degree $\operatorname{deg}(F)$ (sometimes the degree is also denoted by $\left.\operatorname{deg} c_{1}(F)\right)$ as

$$
\operatorname{deg}(F)=\int_{M} \frac{c_{1}(F) \wedge \omega^{n-1}}{\operatorname{vol}(M)}
$$

and the slope $\mu(F)$ as

$$
\mu(F)=\frac{1}{\operatorname{rk}(F)} \cdot \operatorname{deg}(F) .
$$

The number $\mu(F)$ depends only on a cohomology class of $c_{1}(F)$.

Let $F$ be a coherent sheaf on $M$ and $F^{\prime} \subset F$ its subsheaf with $0<\operatorname{rk} F^{\prime}<\operatorname{rk} F$. Then $F^{\prime}$ is called destabilizing subsheaf if $\mu\left(F^{\prime}\right) \geqslant \mu(F)$

A coherent sheaf $F$ is called stable ${ }^{1}$, or $\mu$-stable, if it has no destabilizing subsheaves. A coherent sheaf $F$ is called polystable if it is a direct sum of stable sheaves of the same slope. A coherent sheaf $F$ is called semistable if for all destabilizing subsheaves $F^{\prime} \subset F$ we have $\mu\left(F^{\prime}\right)=\mu(F)$

Let $M$ be a Kähler manifold with a Kähler form $\omega$. Consider the standard Hodge operator on differential forms, $L: \eta \longrightarrow \omega \wedge \eta$. There is also a fiberwiseadjoint Hodge operator $\Lambda$ (see $[\mathrm{GH}]$ ).

\footnotetext{
${ }^{1}$ In the sense of Mumford-Takemoto
} 
Definition 3.4: Let $B$ be a holomorphic bundle over a Kähler manifold $M$ with a holomorphic Hermitian connection $\nabla$ and a curvature $\Theta \in \Lambda^{1,1} \otimes \operatorname{End}(B)$. The Hermitian metric on $B$ and the connection $\nabla$ defined by this metric are called Yang-Mills, or Hermitian-Einstein if

$$
\Lambda(\Theta)=\left.c \cdot \operatorname{Id}\right|_{B},
$$

where $\Lambda$ is a Hodge operator, $c$ a constant, and Id $\left.\right|_{B}$ is the identity endomorphism which is a section of $\operatorname{End}(B)$.

Clearly, the constant $c$ is proportional to the slope of $B$. Throughout this paper, we shall consider only bundles of slope zero. In this case, the Yang-Mills equation can be written simly as $\Lambda(\Theta)=0$.

The following fundamental theorem provides examples of Yang-Mills bundles.

Theorem 3.5: (Uhlenbeck-Yau) Let B be a holomorphic bundle over a compact Kähler manifold. Then $B$ admits a Hermitian Yang-Mills connection if and only if it is polystable. Moreover, the Yang-Mills connection is unique, if it exists.

Proof: [UY].

Remark 3.6: It is easy to see that a connection is Yang-Mills if and only if its curvature is harmonic.

Proposition 3.7: Let $M$ be a hyperkähler manifold, $L$ an induced complex structure and $B$ be a complex vector bundle over $(M, L)$. Then every hyperholomorphic connection $\nabla$ in $B$ is Yang-Mills and satisfies $\Lambda(\Theta)=0$, where $\Theta$ is a curvature of $\nabla$.

Proof: We use the definition of a hyperholomorphic connection as one with $S U(2)$-invariant curvature. Then Proposition 3.7 follows from the following elementary observation

Lemma 3.8: Let $\Theta \in \Lambda^{2}(M)$ be a $S U(2)$-invariant differential 2-form on $M$. Then $\Lambda_{L}(\Theta)=0$ for each induced complex structure $L .^{2}$

Proof: This is Lemma 2.1 of [V0].

\footnotetext{
${ }^{2} \mathrm{By} \Lambda_{L}$ we understand the Hodge operator $\Lambda$ associated with the Kähler complex structure $L$.
} 
Let $M$ be a compact hyperkähler manifold, and $I$ an induced complex structure. For any stable holomorphic bundle on $(M, I)$ there exists a unique Hermitian Yang-Mills connection which, for some bundles, turns out to be hyperholomorphic. It is possible to tell exactly when this happens.

Theorem 3.9: Let $B$ be a polystable holomorphic bundle over $(M, I)$, where $M$ is a hyperkähler manifold and $I$ is an induced complex structure over $M$. Then $B$ admits a hyperholomorphic connection if and only if it is polystable and the first two Chern classes $c_{1}(B)$ and $c_{2}(B)$ are $S U(2)$-invariant. ${ }^{3}$

Proof: This is Theorem 2.5 of [V0].

Definition 3.10: Let $M$ be a compact hyperkähler manifold, $I$ an induced complex structure, and $B$ a polystable bundle on $(M, I)$. We say that $B$ is hyperholomorphic if $B$ admits a hyperholomorphic connection; equivalently, $B$ is hyperholomorphic if the first two Chern classes $c_{1}(B)$ and $c_{2}(B)$ are $S U(2)$ invariant.

From the definition of generic induced complex structures, we immediately obtain the following corollary

Corollary 3.11: Let $M$ be a compact hyperkähler manifold, $I$ an induced complex structure, which is generic in the sense of Definition 2.8, and $B$ a stable bundle over $(M, I)$. Then $B$ is hyperholomorphic.

\section{Generic complex structures ON HYPERKÄHLER MANIFOLDS}

Let $M$ be a compact hyperkähler manifold. In this Section, we follow the arguments of [V2] (see also [V4]), which were used to establish the equivalence between various algebro-geometric structures on $(M, I)$ and $(M, J)$, where $I$ and $J$ are generic complex structures on $M$ induced by a hyperkähler structure in the same connected component of the moduli space.

Given a complex structure $I$ on $M$, let $\mathcal{I}: H^{i}(M) \longrightarrow H^{i}(M)$ be the operator mapping a $(p, q)$-class $\eta$ to $\sqrt{-1}(p-q) \eta$. This gives a $U(1)$-action $\mathfrak{u}_{I}$ on $H^{i}(M)$.

Remark 4.1: Clearly, a form is of type $(p, p)$ with respect to $I$ if and only if it is $\mathfrak{u}_{I}$-invariant.

\footnotetext{
${ }^{3}$ We use Lemma 2.3 to speak of action of $S U(2)$ in cohomology of $M$.
} 
Let $\mathfrak{g}_{0} \subset \operatorname{End}\left(H^{*}(M)\right)$ be the Lie algebra generated by $\mathfrak{u}_{I}$, for all complex structures $I$ which are compatible with some hyperkähler structure on $M$. In [V2], this Lie algebra was computed explicitly, for $M$ with $h^{1}(M)=0, h^{2,0}(M)=1$ (such hyperkähler manifolds are called irreducible, or simple). We have shown that $\mathfrak{g}_{0} \cong \mathfrak{s o}\left(H^{2}(M), h\right)$, where $h$ is a certain non-degenerate symmetric form on $H^{2}(M)$, called the Bogomolov-Beauville form.

By Remark 4.1, a cohomology class $\eta$ is $\mathfrak{g}_{0}$-invariant if and only if $\eta$ is invariant with respect to all complex structures $I$ induced by some hyperkähler structure. For any cohomology class $\eta$ which is not $\mathfrak{g}_{0}$-invariant, let $C_{\eta}$ be the set of all complex structures $I$ which satisfy $\mathfrak{u}_{I}(\eta)=0$. Clearly, $C_{\eta}$ is a closed complex analytic subset in the moduli $\mathcal{M}$ of complex structures on $M$.

Let $S \subset H^{*}(M, \mathbb{Z})$ be the set of all cohomology classes which are not $\mathfrak{g}_{0^{-}}$ invariant, and $C_{S}$ the union of $C_{\eta}$ for all $\eta \in S$. By definition, $C_{S}$ is a countable union of complex subvarieties of positive codimension. Therefore, it has measure zero, and its complement is dense in the moduli space $\mathcal{M}$. We obtain the following definition of generic ([V4]).

Definition 4.2: Let $M$ be a compact manifold of hyperkähler type, and $I$ a complex structure which is compatible with some hyperkähler structure. Consider the Lie algebra $\mathfrak{g}_{0} \subset \operatorname{End}\left(H^{*}(M)\right)$ defined above. Assume that all integer $(p, p)$ classes on $(M, I)$ are $\mathfrak{g}_{0}$-invariant. Then $I$ is called Mumford-Tate generic.

Remark 4.3: When $M$ is a $\mathrm{K} 3$ surface or a torus, $M$ is Mumford-Tate generic if and only if $M$ does not have non-trivial integer $(p, p)$-cycles.

The following claim is clear from the definition.

Claim 4.4: Let $M$ be a hyperkähler manifold, and $I$ an induced complex structure. Assume that $I$ is Mumford-Tate generic. Then $I$ is generic, in the sense of Definition 2.8.

The following theorem is proven in [V2] (see also [V4]).

Theorem 4.5: Let $M$ be a compact hyperkähler manifold, and $I, I^{\prime}$ complex structures of Kähler type which belong to the same connected component of moduli space. Then there exists a sequence $\mathcal{H}_{1}, \mathcal{H}_{2}, \ldots \mathcal{H}_{n}$ of hyperkähler structures, and a set $I_{0}=I, I_{2}, I_{3}, \ldots, I_{n+1}=I^{\prime}$ of complex structures on $M$, such that all $I_{k}$ are Mumford-Tate generic, and the hyperkähler structure $\mathcal{H}_{i}$ induces the complex structures $I_{i}, I_{i-1}$. 


\section{Category of Reflexive sheaves}

In this Section we relate several classic results dealing with reflexive sheaves and their singularities. The proofs and further details can be found e.g. in [OSS].

Definition 5.1: Let $X$ be a complex manifold, and $F$ a coherent sheaf on $X$. Consider the sheaf $F^{*}:=\mathcal{H} m_{\mathcal{O}_{X}}\left(F, \mathcal{O}_{X}\right)$. There is a natural functorial map $\rho_{F}: F \longrightarrow F^{* *}$. The sheaf $F^{* *}$ is called a reflexive hull, or reflexization, of $F$. The sheaf $F$ is called reflexive if the map $\rho_{F}: F \longrightarrow F^{* *}$ is an isomorphism.

Remark 5.2: For all coherent sheaves $F$, the map $\rho_{F^{*}}: F^{*} \longrightarrow F^{* * *}$ is an isomorphism ([OSS], Ch. II, the proof of Lemma 1.1.12). Therefore, a reflexive hull of a sheaf is always reflexive.

Reflexive hull can be obtained by restricting to an open subset and taking the pushforward.

Lemma 5.3: Let $X$ be a complex manifold, $F$ a coherent sheaf on $X, Z$ a closed analytic subvariety, $\operatorname{codim} Z \geqslant 2$, and $j:(X \backslash Z) \hookrightarrow X$ the natural embedding. Assume that the pullback $j^{*} F$ is reflexive on $(X \backslash Z)$. Then the pushforward $j_{*} j^{*} F$ is also reflexive.

Proof: This is [OSS], Ch. II, Lemma 1.1.12.

Definition 5.4: Let $F$ be a coherent sheaf on a complex manifold. For any analytic subvariety $Z \subset X$, denote by $j:(X \backslash Z) \hookrightarrow X$ the natural embedding. Consider the standard sheaf morphism $F \stackrel{\varphi}{\longrightarrow} j_{*} j^{*} F$. The sheaf $F$ is called normal if $\varphi$ is an isomorphism, for all $\operatorname{codim} Z \geqslant 2$.

From Lemma 5.3, the following statement is clear.

Lemma 5.5: ([OSS]) Let $F$ be a coherent torsion-free sheaf on a complex manifold. Then $F$ is normal if and only if $F$ is reflexive.

This leads to the following definition

Definition 5.6: Let $X$ be a complex manifold without subvarieties of codimension 1 . The category of reflexive sheaves on $X$ is defined as

$$
\mathcal{C}^{r}(X):=\underset{\lim }{\longrightarrow} \mathcal{C}(X \backslash Z),
$$

where $\mathcal{C}(X \backslash Z)$ is the category of locally free coherent sheaves on $X \backslash Z$ which can be extended to coherent sheaves on $X$, and the limit is taken over all increasing 
sequences of closed subvarieties

$$
Z \subset X, \quad \operatorname{codim}_{X} Z \geqslant 2
$$

Lemma 5.7: Let $F$ be a reflexive sheaf on $M$, and $X$ its singular set. Then $\operatorname{codim}_{M} X \geqslant 3$

Proof: This is [OSS], Ch. II, 1.1.10.

\section{Twistor FORMALISM AND COHOMOLOGY}

6.1. Twistor spaces. Let $M$ be a hyperkähler manifold. We identify the 2dimensional sphere $S^{2}$ with the set of all quaternions $J$ with $J^{2}=-1$. Consider the product manifold $X=M \times S^{2}$. For every point $x=m \times J \in X=M \times S^{2}$ the tangent space $T_{x} X$ is canonically decomposed $T_{x} X=T_{m} M \oplus T_{J} S^{2}$. Identifying $S^{2}$ with $\mathbb{C} P^{1}$ in a standard fashion, we may assume that $S^{2}$ is equipped with a natural complex structure. Let $I_{S^{2}}: T_{J} S^{2} \longrightarrow T_{J} S^{2}$ be the complex structure operator. Let $I_{M}: T_{m} M \longrightarrow T_{m} M$ be the complex structure on $M$ induced by $J \in S^{2} \subset \mathbb{H}$.

The operator $I_{x}=I_{M} \oplus I_{J}: T_{M} X \longrightarrow T_{x} X$ satisfies $I_{x} \circ I_{x}=-1$. It depends smoothly on the point $x$, hence defines an almost complex structure on $X$. This almost complex structure is known to be integrable (see [Sal]).

Definition 6.1: The complex manifold $\left(X, I_{x}\right)$ is called the twistor space for the hyperkähler manifold $M$, denoted by $\operatorname{Tw}(M)$. This manifold is equipped with a real analytic projection $\sigma: \operatorname{Tw}(M) \longrightarrow M$ and a complex analytic projection $\pi: \operatorname{Tw}(M) \longrightarrow \mathbb{C} P^{1}$.

For any $I \in \mathbb{C} P^{1}$, consider the complex submanifold $\pi^{-1}(I) \subset \operatorname{Tw}(M)$. Clearly, $\pi^{-1}(I)$ is naturally isomorphic to $(M, I)$. Further on, we shall consider $(M, I)$ as a submanifold in $\operatorname{Tw}(M)$.

6.2. Twistor formalism for vector bundles. Let $M$ be a hyperkähler manifold, and $\operatorname{Tw}(M)=M \times \mathbb{C} P^{1}$ its twistor space, equipped with a natural (nonholomorphic) projection $\sigma: \operatorname{Tw}(M) \longrightarrow M$. Given a bundle $B$ with a connection $\nabla$, we can lift $\nabla$ to the pullback $\sigma^{*} B$ to obtain a vector bundle $\left(\sigma^{*} B, \sigma^{*} \nabla\right)$ with 
connection. Take a $(0,1)$-part of $\sigma^{*} \nabla$. By a Newlander-Nirenberg theorem (Theorem 3.1), the operator $\left(\sigma^{*} \nabla\right)^{(0,1)}$ defines a holomorphic structure on $\sigma^{*} B$ if and only if $\left(\left(\sigma^{*} \nabla\right)^{(0,1)}\right)^{2}=0$.

In $[\mathrm{KV}]$, it is shown that this is equivalent to $S U(2)$-invariance of the curvature of the connection $\nabla$.

We obtain a functor (twistor transform)

$$
\sigma^{*}:(B, \nabla) \longrightarrow\left(\sigma^{*} B,\left(\sigma^{*} \nabla\right)^{1,0}\right) .
$$

It turns out that this functor is invertible: the connection $\nabla$ can be reconstructed from the holomorphic structure operator $\left(\sigma^{*} \nabla\right)^{(0,1)}$. The following theorem was proven in $[\mathrm{KV}]$.

Theorem 6.2: The functor (6.1) gives an equivalence of the following categories

(i): Vector bundles $B$ on $M$ with connection $\nabla$ whose curvature

$$
\Theta \in \Lambda^{2}(M) \otimes \text { End } B
$$

is an $S U(2)$-invariant 2 -form on $M$.

(ii): Holomorphic vector bundles $H$ on $\operatorname{Tw}(M)$ such that for all $x \in M$, the restriction of $H$ to a holomorphic curve $\sigma^{-1}(x) \cong \mathbb{C} P^{1} \subset \mathrm{Tw}(M)$ is trivial, as a holomorphic vector bundle:

$$
\left.H\right|_{\sigma^{-1}(x)} \cong \mathcal{O}_{\mathbb{C} P^{1}}^{\oplus n} .
$$

This equivalence is called the twistor transform.

Assume now that $M$ is compact, and let $I$ be an induced complex structure. Given a polystable bundle $B$ on $(M, I)$, with $c_{1}(B), c_{2}(B) S U(2)$-invariant, we can apply Theorem 3.5 and obtain a unique Yang-Mills connection $\nabla$ on $B$. The curvature of $\nabla$ is $S U(2)$-invariant by Theorem 3.9. Consider its twistor transform

$$
\operatorname{Tw}(B):=\left(\sigma^{*} B,\left(\sigma^{*} \nabla\right)^{(0,1)}\right)
$$

which is defined as in Theorem 6.2. We obtained a functor $B \longrightarrow \operatorname{Tw}(B)$ from the category of hyperholomorphic bundles on $(M, I)$ (Definition 3.10$)$ to the category of holomorphic bundles on $\operatorname{Tw}(M)$. 
6.3. Cohomology of $\operatorname{Tw}(B)$. Let $M$ be a compact hyperkähler manifold, $I$ an induced complex structure, $B$ a hyperholomorphic bundle on $(M, I)$, and $\operatorname{Tw}(B)$ the corresponding bundle on a twistor space. Further on in this paper, we construct a holomorphic bundle on a twistor space starting from a semistable bundle on $(M, I)$. For this purpose, we need to relate the cohomology of $\operatorname{Tw}(B)$ and the cohomology of $B$.

Proposition 6.3: Let $M$ be a compact hyperkähler manifold,

$$
\pi: \operatorname{Tw}(M) \longrightarrow \mathbb{C} P^{1}
$$

its twistor space, $L$ an induced complex structure, and $B$ a hyperholomorphic bundle on $(M, L)$. Consider the corresponding bundle $\operatorname{Tw}(B)$ on $\operatorname{Tw}(M)$. Let $R^{i} \pi_{*}(\operatorname{Tw}(B))$ be the higher direct image of $\operatorname{Tw}(B)$, considered as a coherent sheaf on $\mathbb{C} P^{1}$. Then

$$
R^{i} \pi_{*} \operatorname{Tw}(B) \cong \mathcal{O}_{\mathbb{C} P^{1}}(i) \otimes_{\mathbb{C}} H^{i}(B),
$$

where $H^{i}(B)$ is the $i$-th cohomology of $B$ on $(M, L)$.

Proof: Essentially, Proposition 6.3 is proven in [V0].

Consider an induced complex structure $L^{\prime}$. Restricting $\operatorname{Tw}(B)$ to

$$
\left(M, L^{\prime}\right) \subset \operatorname{Tw}(M),
$$

we obtain a holomorphic vector bundle $B_{L^{\prime}}$. Denote its cohomology by $H^{i}\left(B_{L^{\prime}}\right)$. In [V0] it was proven that $H^{i}\left(B_{L^{\prime}}\right)$ is (non-canonically) isomorphic to $H^{i}(B)$. This implies that $R^{i} \pi_{*} \operatorname{Tw}(B)$ is a holomorphic vector bundle, of rank $\operatorname{dim} H^{i}(B)$. By Grothendieck's theorem, $R^{i} \pi_{*} \operatorname{Tw}(B)$ is a direct sum of several copies of $\mathcal{O}(i)$, for various $i$. We need only to show that $R^{i} \pi_{*} \operatorname{Tw}(B)$ is a direct sum of several copies of $\mathcal{O}(i)$.

To prove this, we need to repeat the argument of [V0], taking care of the dependency of $H^{i}\left(B_{L^{\prime}}\right)$ from the induced complex structure $L^{\prime}$.

We say that an $S U(2)$-representation $V$ has weight $i$ if $V$ is generated by the highest weight vectors of weight $i$. An element $v \in V$ has weight $i$ if $v$ belongs to a subrepresentation of weight $i$.

Consider the space $\Lambda_{+}^{i}(M) \subset \Lambda^{i}(M)$ consisting of all $i$-forms which have weight $i$ (by multiplicativity of weight, an $i$-form has weight $\leqslant i$ ). The space $\Lambda_{+}^{i}(M)$ is compatible with the Hodge grading, that is, it admits the Hodge decomposition 
for every induced complex structure $I$

$$
\Lambda_{+}^{i}(M)=\oplus_{p+q=i} \Lambda_{I,+}^{p, q}(M) .
$$

In [V5], the space $\Lambda_{+}^{i}(M) \otimes B$ was studied in great detail. We have shown that $\Lambda_{+}^{i}(M) \otimes B$ admits a natural $S U(2)$-invariant Laplacian $\Delta_{+}$, whenever $B$ is equipped with a hyperholomorphic connection. Moreover, $\Delta_{+}$is compatible with the Hodge decomposition, and for all induced complex structures $I$ we have a canonical identification

$$
\mathcal{H}_{+, I}^{0, p}(M, B)=H^{p}\left(B_{I}\right)
$$

where $\mathcal{H}_{+, I}^{0, p}(M, B)$ is the space of $\Delta_{+}$-harmonic forms of type $(0, p)$, and $H^{p}\left(B_{I}\right)$ the space of cohomology of $B$ considered as a holomorphic bundle on $(M, I)$.

Let

$$
\mathcal{W}:=\mathcal{H}_{+}^{p}(M, B) \otimes_{\mathbb{C}} \mathcal{O}_{\mathbb{C} P^{1}}
$$

be the trivial vector bundle with the fiber $\mathcal{H}_{+}^{p}(M, B)$. Using the Hodge decomposition (6.2) on the space of $\Delta_{+}$-harmonic forms $\mathcal{H}_{+}^{p}(M, B)$, we can decompose $\mathcal{H}_{+}^{p}(M, B) \otimes_{\mathbb{C}} \mathcal{O}_{\mathbb{C} P^{1}}$ onto its Hodge components ${ }^{1}$

$$
\left.\mathcal{W}\right|_{I}=\oplus_{p+q=i} \mathcal{H}_{+, I}^{p, q}(M, B)
$$

By (6.3), the bundle $R^{p} \pi_{*} \operatorname{Tw}(B)$ is identified with the $(0, p)$-part $\mathcal{W}^{0, p}$ of the trivial vector bundle $\mathcal{W}=\mathcal{H}_{+}^{p}(M, B) \otimes_{\mathbb{C}} \mathcal{O}_{\mathbb{C} P^{1}}$.

Consider the natural embedding $\Xi$ of $\mathcal{H}_{+, I}^{0, p}(M, B)$ to the space

$$
\Lambda_{\pi}^{0, p}(\operatorname{Tw}(M)) \otimes \operatorname{Tw}(B)
$$

of relative $\operatorname{Tw}(B)$-valued $(0, p)$-forms on the space $\operatorname{Tw}(M)$. On $(0, p)$-forms, the $S U(2)$-invariant Laplace operator $\Delta_{+}$is equal to the usual Dolbeault Laplacian

$$
\Delta_{\bar{\partial}}=\partial \partial^{*}+\partial^{*} \partial .
$$

Therefore, $\Xi$ is a holomorphic embedding, and we can reconstruct the holomorphic structure on $R^{p} \pi_{*} \operatorname{Tw}(B)$ from that on $\Lambda_{\pi}^{0, p}(\operatorname{Tw}(M)) \otimes \operatorname{Tw}(B)$.

Since the holomorphic structure on $R^{p} \pi_{*} \operatorname{Tw}(B)=\mathcal{W}^{0, p}$ is compatible with the standard embedding

$$
\Xi: \mathcal{H}_{+, I}^{0, p}(M, B) \longrightarrow \Lambda_{\pi}^{0, p}(\operatorname{Tw}(M)) \otimes \operatorname{Tw}(B),
$$

\footnotetext{
${ }^{1}$ This decomposition is not holomorphic. In fact, the Hodge decomposition (6.4) defines a non-polarized variation of Hodge structures on $\mathcal{H}_{+}^{p}(M, B) \otimes_{\mathbb{C}} \mathcal{O}_{\mathbb{C} P^{1}}$.
} 
the holomorphic structure on $R^{p} \pi_{*} \operatorname{Tw}(B)=\mathcal{W}^{0, p}$ is inherited from $\mathcal{W}$, as follows. Take a section $Y$ of $\mathcal{W}^{0, p}$, and let $\bar{\partial}_{\mathcal{W}} Y, \bar{\partial}_{\mathcal{W}^{0, p}} Y$ be the holomorphic structure operator on $\mathcal{W}, \mathcal{W}^{0, p}$ applied to $Y$. Then $\bar{\partial}_{\mathcal{W}^{0, p}} Y$ is equal to the orthogonal projection of

$$
\bar{\partial}_{\mathcal{W}} Y \subset \Lambda^{(0,1)} \mathbb{C} P^{1} \otimes \mathcal{W}
$$

to $\mathcal{W}^{0, p} \subset \mathcal{W}$.

Using the natural $S U(2)$-action on $\mathbb{C} P^{1}$ and $\mathcal{H}_{+}^{p}(M, B)$, we may consider $\mathcal{W}$ as an $S U(2)$-equivariant bundle. Let $I$ be an arbitrary induced complex structure, and denote by $\mathcal{I}$ the Lie algebra element associated with $I^{2}$. Then $\left.\mathcal{W}^{0, p}\right|_{I}$ is by definition the space of all vectors $\left.v \in \mathcal{W}\right|_{I}$ which satisfy $\mathcal{I}(v)=-p \sqrt{-1} v$.

We arrive at the following situation. Let $W$ be an irreducible $S U(2)$-representation of weight $p$, and

$$
\mathcal{W}=W \otimes_{\mathbb{C}} \mathcal{O}_{\mathbb{C} P}
$$

a trivial bundle with an $S U(2)$-equivariant action, induced by the $S U(2)$-action on $W$ and $\mathbb{C} P^{1}$. Consider a $C^{\infty}$-subbundle $\mathcal{W}^{0, p} \subset \mathcal{W}$ of all vectors $v \in \mathcal{W}_{\left.\right|_{I}}$ which satisfy

$$
\mathcal{I}(v)=-p \sqrt{-1} v
$$

and let the holomorphic structure on $\mathcal{W}^{0, p}$ act as in (6.5). Then Proposition 6.3 is implied by the following claim, which is a version of Borel-Bott-Weyl theorem for the group $S L(2)$.

Claim 6.4: In the above assumptions, the bundle $\mathcal{W}^{0, p}$ is isomorphic to a sum of several copies of $\mathcal{O}(p)$.

Proof: Clearly, it suffices to prove Claim 6.4 when $W$ is an irreducible representation of weight $p$. In this case, $\mathcal{W}^{0, p}$ is obviously a line bundle.

Assume that $V$ is an irreducible representation of weight 1 , and let $\mathcal{V}^{0,1}$ be the line bundle constructed as above from $V$. From (6.6) one can easily see that

$$
\mathcal{W}^{0, p} \cong\left(V^{0,1}\right)^{\otimes p}
$$

By (6.7), it suffices to prove Claim 6.4 for $p=1$, and $W$ 2-dimensional.

\footnotetext{
${ }^{2}$ The action of $\mathcal{I}$ induces the Hodge decomposition associated with $I$ in a standard way.
} 
Consider $\mathbb{C} P^{1}$ as a space of 1 -dimensional subspaces in $W$. Using the action of $S U(2)$ on $W$, we equip the bunle $\mathcal{O}(1)$ of linear forms on these subspaces with a natural $S U(2)$-equivariant structure. Consider a point $(1,0) \in \mathbb{C} P^{1}$ corresponding to an induced complex structure $I$. By definition, the Lie algebra element $\mathcal{I}$ acts on the line $(\mathbb{C}, 0)$ as $\sqrt{-1}$, hence it acts on its dual space as $-\sqrt{-1}$. Since a $G$-equivariant bundle over a manifold with a homogeneous $G$-action is uniquely determined by the action of the stabilizer of a point, we obtain that $\mathcal{O}(1)$ is isomorphic to $\mathcal{W}^{0,1}$ as an equivariant bundle. Therefore, $c_{1}\left(W^{0,1}\right)=c_{1}(\mathcal{O}(1))=1$, and these line bundles are isomorphic as holomorphic bundles as well. Claim 6.4 is proven. We proved Proposition 6.3.

\section{LOCAL GEOMETRY OF THE TWISTOR SPACE}

7.1. Twistor lines and local geometry. Consider the standard holomorphic projection $\pi: \operatorname{Tw}(M) \longrightarrow \mathbb{C} P^{1}$. To study $\operatorname{Tw}(M)$, we use the space of holomorphic sections

$$
s: \mathbb{C} P^{1} \longrightarrow \operatorname{Tw}(M)
$$

of $\pi$. Given $x \in M$, we have a tautological section $s_{x}$ mapping $I \in \mathbb{C} P^{1}$ to $(x, I) \in \operatorname{Tw}(M)=M \times \mathbb{C} P^{1}$. Such sections are called the horizontal sections of $\pi$, or horizontal twistor lines. A normal bundle to $s$ is isomorphic to $\mathcal{O}(1)^{\oplus n}$ ([HKLR]). By deformation theory, this implies the following.

Given two distinct points $I_{1}, I_{2}$ on $\mathbb{C} P^{1}$, and two sufficiently close points $x_{1}, x_{2} \in M$, there exists a unique section $s: \mathbb{C} P^{1} \longrightarrow \mathrm{Tw}(M)$ which is close to a horizontal section and passes through $\left(I_{1}, x_{1}\right)$ and $\left(I_{2}, x_{2}\right)$ (see [V3], (7.2) for a more rigorous version of this statement).

On an intuitive level, the twistor space is very close to a projective space; indeed, for $M=\mathbb{H}, \operatorname{Tw}(M)$ is isomorphic to $\mathbb{C} P^{3}$ without a line.

Fix a point $x_{0} \in M$, and the correspoinding point in twistor space $\left(I, x_{0}\right) \in$ $\operatorname{Tw}(M)$. We shall study the twistor sections passing through $\left(I, x_{0}\right) \in \operatorname{Tw}(M)$ in a neighbourhood of the horisontal section $s_{x_{0}}$.

For every $L, L^{\prime} \in \mathbb{C} P^{1}$, let $\mathcal{O}_{x_{0}, L}, \mathcal{O}_{x_{0}, L^{\prime}}$ be the ring of germs of complex analytic functions on $(M, L)$ and $\left(M, L^{\prime}\right)$ in $x_{0}$. In [V3], 5.3, we constructed a 
natural isomorphism

$$
\Psi_{I}\left(L, L^{\prime}\right): \mathcal{O}_{x_{0}, L} \longrightarrow \mathcal{O}_{x_{0}, L^{\prime}}
$$

associated with the local map $\widetilde{\Psi}_{I}:\left(M, L^{\prime}\right) \longrightarrow(M, L)$ defined in an appropriate neighbourhood of $x_{0}$ as follows. ${ }^{1}$

Consider a point $\left(m, L^{\prime}\right)$ close to $\left(x_{0}, L^{\prime}\right)$. By (7.1), there exists twistor section $s: \mathbb{C} P^{1} \longrightarrow \operatorname{Tw}(M)$ passing through $\left(m, L^{\prime}\right)$ and $\left(x_{0}, I\right)$ and close to $s_{x_{0}}=$ $\left\{x_{0}\right\} \times \mathbb{C} P^{1}$. This section is unique in a small neighbourhood of $\left\{x_{0}\right\} \times \mathbb{C} P^{1}$. We define $\widetilde{\Psi}_{I}(m):=s(L) \in(M, L)$ (see Figure 1). This map is holomorphic and invertible in a sufficiently small neighbourhood of $\left(x_{0}, L^{\prime}\right)$ (q.v. [V3], Definition 7.6).

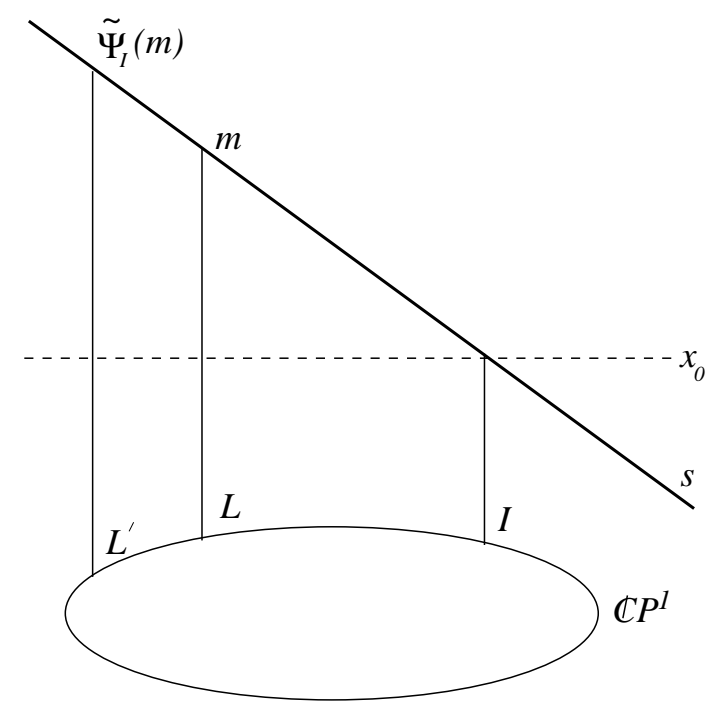

Figure 1. Constructing $\widetilde{\Psi}_{I}$ with twistor sections

Let now $B$ be a bundle on $\operatorname{Tw}(M)$ obtained from the twistor transform (Theorem 6.2). This means that the restriction of $B$ to any horizontal twistor section $s_{x}$ is trivial. Clearly, a small deformation of a trivial bundle on $\mathbb{C} P^{1}$ is again trivial. Therefore, the restriction of $B$ to a twistor section close to $s_{x}$ is trivial as well.

\footnotetext{
${ }^{1}$ In [V3], 5.3 this map is obtained as a composition of the maps $i_{L}$ and $e_{L^{\prime}}$ defined algebraically on the appropriate spaces of germs. A geometric treatment of the same maps is given in [V3], Section 7-8.
} 
This allows one to extend the map $\Psi_{I}\left(L, L^{\prime}\right): \mathcal{O}_{x_{0}, L} \longrightarrow \mathcal{O}_{x_{0}, L^{\prime}}$ to the space of germs of holomorphic sections of $B$ (see [V4] for a more detailed construction). We obtain an isomorphism

$$
\Psi_{I}\left(L, L^{\prime}, B\right): B_{x_{0}, L} \longrightarrow B_{x_{0}, L^{\prime}}
$$

where $B_{x_{0}, L}$ and $B_{x_{0}, L^{\prime}}$ are spaces of germs of $\left.B\right|_{(M, L)}$ and $\left.B\right|_{\left(M, L^{\prime}\right)}$ :

$$
B_{x_{0}, L}=\left.\mathcal{O}_{x_{0}, L} \otimes B\right|_{(M, L)} \quad B_{x_{0}, L^{\prime}}=\left.\mathcal{O}_{x_{0}, L^{\prime}} \otimes B\right|_{\left(M, L^{\prime}\right)} .
$$

The isomorphisms $\Psi_{I}\left(L, L^{\prime}\right)$ and $\Psi_{I}\left(L, L^{\prime}, B\right)$ depend on $L, L^{\prime} \in \mathbb{C} P^{1}$ holomorphically (q. v. [V4]).

Consider the ring $\mathcal{O}_{(\operatorname{Tw}(M) \backslash(M, I)), s_{x_{0}}}$ of germs of $\mathcal{O}_{(\operatorname{Tw}(M) \backslash(M, I))}$ in a neighbourhood of

$$
s_{x_{0}} \backslash\left(I, x_{0}\right) \subset \operatorname{Tw}(M) \backslash(M, I) .
$$

Denote the infinitesimal neighbourhood of $s_{x_{0}} \backslash\left(I, x_{0}\right)$ in $\operatorname{Tw}(M) \backslash(M, I)$ by

$$
\operatorname{Tw}(M)_{x_{0}, I}=\operatorname{Spec}\left(\mathcal{O}_{\operatorname{Tw}(M) \backslash(M, I), s_{x_{0}}}\right) .
$$

This space is fibered over $\mathbb{C} P^{1} \backslash I$ with fibers isomorphic to a germ of a smooth complex manifold.

The maps (7.2) produce a canonical trivialization of $\operatorname{Tw}(M)_{x_{0}, I}$ over $\mathbb{C} P^{1} \backslash I$. Denote the trivialization map by

$$
\Phi: \operatorname{Tw}(M)_{x_{0}, I} \longrightarrow S \times\left(\mathbb{C} P^{1} \backslash I\right)
$$

where $S$ is a germ of a complex manifold isomorphic to any of the fibers of the standard projection

$$
\pi: \operatorname{Tw}(M)_{x_{0}, I} \longrightarrow \mathbb{C} P^{1} \backslash I .
$$

Let $\xi: \operatorname{Tw}(M)_{x_{0}, I} \longrightarrow S$ be the composition of $\Phi$ and the projection $S \times$ $\left(\mathbb{C} P^{1} \backslash I\right) \longrightarrow S$. Using the maps $\Psi_{I}\left(L, L^{\prime}, B\right)$ of $(7.3)$, we obtain a trivialization of $\left.B\right|_{\operatorname{Tw}(M)_{x_{0}, I}}$ over $\mathbb{C} P^{1} \backslash I$. More precisely, we obtain a bundle $B_{S}$ over $S$, and a natural isomorphism

$$
\left.B\right|_{\operatorname{Tw}(M)_{x_{0}, I}} \cong \xi^{*} B_{S}
$$


7.2. Local trivialization of the sheaf of differential operators. Let $M$ be a hyperkähler manifold, $\operatorname{dim}_{\mathbb{C}} M=2 n, \pi: \operatorname{Tw}(M) \longrightarrow \mathbb{C} P^{1}$ its twistor space, $x \in M$ a point, $s_{x}:=\{x\} \times \mathbb{C} P^{1} \subset \operatorname{Tw}(M)$ the corresponding rational curve in the twistor space, and $B$ a bundle on $\operatorname{Tw}(M)$ obtained from the twistor transform. By definition, this means that the restriction of $B$ to a curve $s_{x^{\prime}} \subset \operatorname{Tw}(M)$ is a trivial bundle, for all $x^{\prime} \in M .^{2}$ Fix an induced complex structure $I$. In Subsection 7.1, we have constructed a trivialization of $\left.B\right|_{\mathrm{Tw}(M) \backslash(M, I)}$ in a neighbourhood of $s_{x} \backslash I$. In this Subsection we study the asymptotic properties of this trivialization.

Let $\mathcal{O}_{s_{x}}$ be the ring of germs of $\mathcal{O}_{\mathrm{Tw}(M)}$ in a neighbourhood of $s_{x}$. Denote by $\mathfrak{m}_{s_{x}}$ the ideal of all functions vanishing in $s_{x}$, and let $\hat{\mathcal{O}}_{s_{x}}$ be the $\mathfrak{m}_{s_{x}}$-completion of $\mathcal{O}_{s_{x}}$.

We consider $\hat{\mathcal{O}}_{s_{x}}$ with the natural $\mathfrak{m}_{s_{x}}$-adic topology on it. Clearly, we have

$$
\mathfrak{m}_{s_{x}} /\left.\mathfrak{m}_{s_{x}}^{2} \cong \Omega_{\pi}^{1} \operatorname{Tw}(M)\right|_{s_{x}} .
$$

Using e.g. Claim 6.4, it is easy to check that the sheaf of conormal $(1,0)$-vectors to $s_{x}$ is $\mathcal{O}(-1)^{\oplus n}$ :

$$
\left.\Omega_{\pi}^{1} \operatorname{Tw}(M)\right|_{s_{x}} \cong \mathcal{O}(-1)^{\oplus n}
$$

Taking a symmetric power of (7.7), and using (7.6), we obtain

$$
\mathfrak{m}_{s_{x}}^{i} / \mathfrak{m}_{s_{x}}^{i+1} \cong \mathcal{O}(-i) \otimes_{\mathbb{C}} S^{i}\left(\mathbb{C}^{n}\right),
$$

where $S^{i}\left(\mathbb{C}^{n}\right)$ denotes the $i$-th symmetric power of the $n$-dimensional complex space $\mathbb{C}^{n}$.

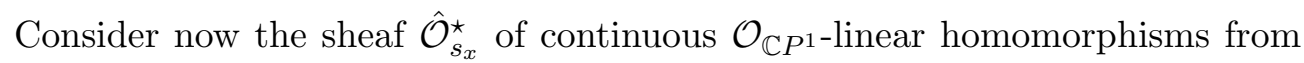
$\hat{\mathcal{O}}_{s_{x}}$ to $\mathcal{O}_{\mathbb{C} P^{1}}$. The sheaf $\hat{\mathcal{O}}_{s_{x}}^{\star}$ has a natural $\mathcal{O}_{\mathrm{Tw}}(M)^{- \text {structure; for any section }}$ $\gamma \in \hat{\mathcal{O}}_{s_{x}}^{\star}, \delta \in \mathcal{O}_{\mathrm{Tw}(M)}, \varepsilon \in \hat{\mathcal{O}}_{s_{x}}$ we write

$$
\delta \cdot \gamma(\varepsilon)=\gamma(\delta \varepsilon)
$$

This gives a structure of quasicoherent sheaf on $\hat{\mathcal{O}}_{s_{x}}^{\star}$.

We consider $\hat{\mathcal{O}}_{s_{x}}^{\star}$ as an infinite-dimensional vector bundle on $\mathbb{C} P^{1}$. The trivialization of $\mathcal{O}_{s_{x}}$ outside $I$ gives a trivialization of $\hat{\mathcal{O}}_{s_{x}}$ outside $I$. However, the bundle $\hat{\mathcal{O}}_{s_{x}}$ is very negative by (7.8), and therefore admits no non-trivial sections. By the same reason, the bundle $\hat{\mathcal{O}}_{s_{x}}^{\star}$ is very positive, hence it admits many

\footnotetext{
${ }^{2}$ Any successive extension of the bundles of form $\operatorname{Tw}\left(B_{i}\right)$, where $B_{i}$ are hyperholomorphic, satisfies this condition.
} 
sections. One should expect that any section of $\left.\hat{\mathcal{O}}_{s_{x}}^{\star}\right|_{\mathbb{C} P^{1} \backslash I}$ compatible with a trivialization can be extended to $\mathbb{C} P^{1}$. The aim of this section is to show that this is indeed so.

Definition 7.1: Given a bundle $F$ on $\mathbb{C} P^{1}$ with a trivialization

$$
\left.F\right|_{\mathbb{C} P^{1} \backslash I} \cong \mathcal{O}_{\mathbb{C} P^{1} \backslash I} \otimes_{\mathbb{C}} \mathbf{F}
$$

and a section $\left.f \in F\right|_{\mathbb{C P}^{1} \backslash I}$, we say that $f$ is compatible with the trivialization if there exists $f \in \mathbf{F}$ such that

$$
f=1 \otimes_{\mathbb{C}} f \in \mathcal{O}_{\mathbb{C} P^{1} \backslash I} \otimes_{\mathbb{C}} \mathbf{F}
$$

Consider the sheaf

$$
B_{s_{x}}^{\star}:=B^{*} \otimes_{\mathcal{O}_{\mathrm{Tw}(M)}} \hat{\mathcal{O}}_{s_{x}}^{\star}
$$

where $B$ is a bundle on $\operatorname{Tw}(M)$ considered in the beginning of this Subsection, and $B^{*}$ its dual. Using the arguments of Subsection 7.1, we obtain a natural trivialization of $B_{s_{x}}^{\star}$ over $\mathbb{C} P^{1} \backslash I$. We study the asymptotical behaviour of this trivialization.

Proposition 7.2: In the above assumptions, let $\left.r \in B_{s_{x}}^{\star}\right|_{\mathbb{C} P^{1} \backslash I}$ be a section which is compatible with the trivialization. Then $r$ can be extended to a section $\widetilde{r}$ of $B_{s_{x}}^{\star}$. Moreover, $\widetilde{r}$ vanish at $I$.

Proof: To prove Proposition 7.2, we interpret $B_{s_{x}}^{\star}$ in terms of differential operators.

Definition 7.3: Let $A$ be an algebra over the ring $B$, and $M, N A$-modules. Following Grothendieck, we define the space $D_{A, B}(M, N) \subset \operatorname{Hom}_{B}(M, N)$ of $B$-linear differential operators from $M$ to $N$ inductively as follows.

An 0-th order differential operator $f \in D_{A, B}^{0}(M, N)$ is an $A$-linear map from $M$ to $N$. An $n$-th order differential operator $\delta: M \longrightarrow N$ is a $B$-linear map such that for all $a \in A$, the commutator $[a, \delta]$ is an $(n-1)$-st order differential operator. The commutator $[a, \delta]: M \longrightarrow N$ is defined as follows:

$$
[a, \delta](m)=\delta(a m)-a \delta(m) .
$$

The following observation is quite elementary. 
Claim 7.4: In the assumptions of Proposition 7.2, the sheaf $B_{s_{x}}^{\star}$ is naturally isomorphic to the sheaf

$$
D_{\mathcal{O}_{\mathrm{Tw}(M), \mathcal{O}}{ }_{\mathbb{C} P}}\left(B, \mathcal{O}_{s_{x}}\right)
$$

of $\mathcal{O}_{\mathbb{C} P^{1}}$-linear differential operators from $B$ to the structure sheaf $\mathcal{O}_{s_{x}}$ of $s_{x} \subset$ $\operatorname{Tw}(M)$, considered as a $\mathcal{O}_{\mathrm{Tw}(M)}$-module.

Proof: Denote by

$$
D^{n}\left(B, \mathcal{O}_{s_{x}}\right) \subset D_{\mathcal{O}_{\mathrm{Tw}(M), \mathcal{O}_{\mathbb{C P}}}}\left(B, \mathcal{O}_{s_{x}}\right)
$$

the sheaf of $n$-th order differential operators. Since

$$
B_{s_{x}}^{\star} \cong \lim _{\longrightarrow} \operatorname{Hom}_{\mathcal{O}_{\mathbb{C} P^{1}}}\left(B / \mathfrak{m}_{s_{x}}^{i} B\right),
$$

to prove Claim 7.4 it suffices to show that $D^{n}\left(B, \mathcal{O}_{s_{x}}\right)$ is dual over $\mathcal{O}_{\mathbb{C} P}$ to $B / \mathfrak{m}_{s_{x}}^{i+1} B$, and that this duality is compatible with the corresponding arrows.

Take $\underline{b} \in B / \mathfrak{m}_{s_{x}}^{n+1} B$, and let $b \in B$ be its representative. Given $\delta \in D^{n}\left(B, \mathcal{O}_{s_{x}}\right)$, we define $\langle\underline{b}, \delta\rangle:=\delta(b)$. Since $\delta$ is of $n$-th order, $\langle\underline{b}, \delta\rangle$ is independent from the choice of $b \in B$. Clearly, for all $\delta \neq 0$ there is $b$ such that $\langle b, \delta\rangle \neq 0$. Comparing the dimensions of $D^{n}\left(B, \mathcal{O}_{s_{x}}\right)$ and $B / \mathfrak{m}_{s_{x}}^{n+1} B$, considered as a finite-dimensional bundles on $\mathbb{C} P^{1}$, we find that this pairing is non-degenerate. We have shown that $D^{n}\left(B, \mathcal{O}_{s_{x}}\right)$ is dual to $B / \mathfrak{m}_{s_{x}}^{i+1} B$. Claim 7.4 is proven.

Now let $\left.D \in D^{n}\left(B, \mathcal{O}_{s_{x}}\right)\right|_{\mathbb{C P}^{1} \backslash I}$ be a differential operator which is compatible with the trivialization. We shall describe $D$ in explicit terms as follows.

Let $L \neq I$ be an arbitrary induced complex structure, and $U_{L}$ the germ of $(M, L)$ at $x \in M$. For any $y \in U_{L}$, let $r_{y}: \mathbb{C} P^{1} \longrightarrow \mathrm{Tw}(M)$ be the unique line close to $s_{x}$ and passing through $(y, L)$ and $x, I \in \operatorname{Tw}(M)$. This gives a map $U_{L} \times \mathbb{C} P^{1} \stackrel{\Xi}{\longrightarrow} \mathrm{Tw}(M),\left(y, L^{\prime}\right) \longrightarrow r_{y}\left(L^{\prime}\right)$ (see Figure 2 ).

Given a differential operator $\underline{D_{1}}:\left.B\right|_{U_{L}} \longrightarrow \mathcal{O}_{U_{L}}$, consider the operator

$$
D_{1}:=\underline{D}_{1} \otimes i d: \Xi^{*}\left(\left.B\right|_{U_{L}}\right) \longrightarrow \mathcal{O}_{U_{L} \times \mathbb{C} P^{1}}
$$

We define the operator

$$
\Xi\left(\underline{D}_{1}\right) \in D_{\mathcal{O}_{\mathrm{Tw}(M), \mathcal{O}} \mathbb{C} P 1}\left(B, \mathcal{O}_{s_{x}}\right)
$$

as follows: given a section $b \in B$, we take the pullback $\Xi^{*}(b)$, apply $D_{1}$ and then restrict to

$$
s_{x}=\{x\} \times \mathbb{C} P^{1} \subset U_{L} \times \mathbb{C} P^{1}=\operatorname{Tw}\left(U_{L}\right) .
$$




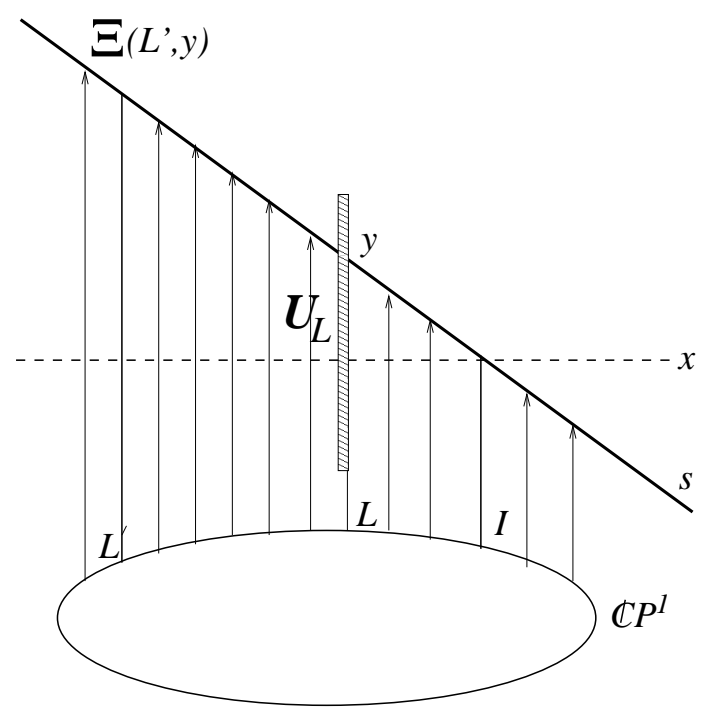

Figure 2. The map $U_{L} \times \mathbb{C} P^{1} \stackrel{\Xi}{\longrightarrow} \operatorname{Tw}(M)$

Tracing back to the definition, we obtain that the differential operator

$$
\left.D \in D_{\mathcal{O}_{\mathrm{Tw}(M), \mathcal{O}_{\mathbb{C} P}}}\left(B, \mathcal{O}_{s_{x}}\right)\right|_{\mathbb{C P}^{1} \backslash I},
$$

is compatible with the trivialization if and only if $D$ is constructed as above: $D=\left.\Xi\left(\underline{D}_{1}\right)\right|_{\mathbb{C} P^{1} \backslash I}$, where where $\underline{D}_{1}$ is a differential operator on $U_{L}$. When $D$ is a first order differential operator, that is, a vector field, this can be illustrated by the following picture (Figure 3).

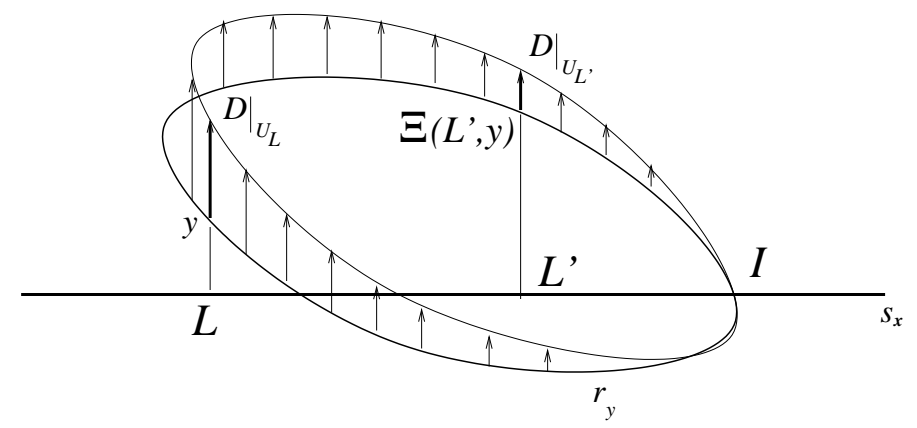

FiguRE 3. First order differential operator trivialized by local twistor geometry

In Figure 3, the little arrows represent the vertical vector field corresponding to $D=\Xi\left(\underline{D}_{1}\right)$. To differentiate a function $f$ along $D$, we look how $r_{y}\left(L^{\prime}\right)$ changes 
as $y$ goes along the vector field $\underline{D}_{1}$ on $U_{L}$. Now Proposition 7.2 is apparent: given a differential operator $\underline{D}_{1}$ on $U_{L}$, and a germ of $B$ around $s_{x} \subset \operatorname{Tw}(M)$, we define $\Xi\left(\underline{D}_{1}\right)(b)$ as above, and the differential operator $\Xi\left(\underline{D}_{1}\right)$ extends from $\mathbb{C} P^{1} \backslash I$ to $\mathbb{C} P^{1}$ in a natural way (in Figure 3 , the vector fields $D$ vanishes at $I$ ). Proposition 7.2 is proven.

7.3. Twistor space and cohomology with support. Given a coherent sheaf $F$ on $X$ and a finite set $A \subset X$, let $\mathcal{H}_{A}^{i}(F)$ be the sheaf of cohomology of $F$ with support in $A$. Further on, we shall need the following characterization of the cohomology with support.

Proposition 7.5: (Grothendieck's local duality) Let $X$ be a compact complex manifold, $A \subset X$ a finite subset, and $B$ a holomorphic bundle on $X$. Then $\mathcal{H}_{A}^{i}(B)=0$ for all $i \neq \operatorname{dim} X$. For $i=\operatorname{dim} X$ the sheaf $\mathcal{H}_{A}^{i}(B)$ can be expressed as follows. Take the completion $\hat{\mathcal{O}}_{X, A}$ of $\mathcal{O}_{X}$ in $A$-adic topology. Consider the dual space $\operatorname{Hom}_{\mathbb{C}}\left(\hat{\mathcal{O}}_{X, A}, \mathbb{C}\right)$ of continuous $\mathbb{C}$-linear maps from $\hat{\mathcal{O}}_{X, A}$ to $\mathbb{C}$, equipped with a natural $\mathcal{O}_{X}$-action. Let $D_{A}:=\operatorname{Hom}_{\mathbb{C}}\left(\hat{\mathcal{O}}_{X, A}, \mathbb{C}\right) \otimes_{\mathcal{O}_{X}} K_{X}$ be the product of $\operatorname{Hom}_{\mathbb{C}}\left(\hat{\mathcal{O}}_{X, A}, \mathbb{C}\right)$ with the canonical sheaf. Then $\mathcal{H}_{A}^{i}(B)$ is naturally isomorphic to $D_{A}$.

Proof: See $[\mathrm{GH}], \S 4$, Example 3.

From Proposition 7.5 we can easily obtain the following corollary.

Corollary 7.6: Let $X$ be a compact complex manifold, $F$ a coherent sheaf on $X$ and $Z \subset X$ a non-empty complex analytic subset. Then $H^{n}\left(X \backslash Z,\left.F\right|_{X \backslash Z}\right)=0$, for $n \geqslant \operatorname{dim}_{\mathbb{C}} X$.

Proof: See [GH], Theorem 6.9.

Remark 7.7: The statement of Corollary 7.6 is often formulated as follows: one says that $X \backslash Z$ has cohomological dimension at most $\operatorname{dim} X-1$.

Let $M$ be a compact hyperkähler manifold, $I$ an induced complex structure, $A$ a finite set, and $B$ a hyperholomorphic bundle on $M$. We study the cohomology of the holomorphic bundle $\left.\operatorname{Tw}(B)\right|_{\operatorname{Tw}(M \backslash A)}$. Consider $B$ as a holomorphic bundle on $(M, I)$. Let $j:(M \backslash A) \hookrightarrow M$ be the natural embedding. For an acyclic sheaf $\mathcal{F}$, consider the exact sequence

$$
0 \longrightarrow H_{A}^{0}(\mathcal{F}) \longrightarrow F \longrightarrow j^{*} \mathcal{F} \longrightarrow 0
$$


The corresponding long exact sequence gives

$$
H_{A}^{1}(B) \longrightarrow H^{1}(B) \longrightarrow H^{1}\left(j^{*} B\right) \longrightarrow H_{A}^{2}(B)
$$

For $\operatorname{dim}_{\mathbb{C}} M>2$, we have $H_{A}^{2}(B)=H_{A}^{1}(B)=0$ (Proposition 7.5). Then, (7.9) implies $H^{1}\left(j^{*} B\right) \cong H^{1}(B)$. For $\operatorname{dim}_{\mathbb{C}} M=2$, the situation is different. We have a long exact sequence

$$
0 \longrightarrow H^{1}(B) \longrightarrow H^{1}\left(j^{*} B\right) \longrightarrow H_{A}^{2}(B) \longrightarrow H^{2}(B) \longrightarrow H^{2}\left(j^{*} B\right) .
$$

However, the cohomological dimension of $M \backslash A$ is $\leqslant 1$ by Corollary 7.6, hence $H^{2}\left(j^{*} B\right)=0$. We obtain the following result.

Corollary 7.8: Let $X$ be a complex surface, $B$ a holomorphic vector bundle, and $A$ a non-empty finite set. Then we have an exact sequence

$$
0 \longrightarrow H^{1}(B) \longrightarrow H^{1}\left(j^{*} B\right) \longrightarrow H_{A}^{2}(B) \longrightarrow H^{2}(B) \longrightarrow 0 .
$$

The main result of this Subsection is the following

Proposition 7.9: Let $M$ be a hyperkähler manifold of real dimension 4, $\mathrm{Tw}(M) \stackrel{\pi}{\longrightarrow} \mathbb{C} P^{1}$ its twistor space, $I$ an induced complex structure, $A \subset M$ a finite set, and $B$ a hyperholomorphic bundle on $M$. Consider $B$ as a holomorphic bundle on $(M, I)$. Let $\operatorname{Tw}(B)$ be the twistor transform of $B$. Denote by $\mathrm{Tw}_{A}(B)$ the restriction of $\operatorname{Tw}(B)$ to $\operatorname{Tw}(M \backslash A)$. Then

$$
H^{i}\left(\operatorname{Tw}_{A}(B)\right)=0 \quad \text { for } \quad i>1 .
$$

Moreover, we have an exact sequence of sheaves on $\mathbb{C} P^{1}$

$$
\begin{aligned}
0 \longrightarrow & H^{1}(B) \otimes_{\mathbb{C}} \mathcal{O}(1) \longrightarrow R^{1} \pi_{*} \operatorname{Tw}_{A}(B) \\
& \longrightarrow \bigoplus_{x_{i} \in A} \operatorname{Hom}_{\mathbb{C}}\left(\hat{\mathcal{O}}_{s_{x_{i}}}, \mathbb{C}\right) \otimes_{\mathcal{O}_{\mathrm{Tw}(M)}} \operatorname{Tw}(B) \otimes_{\mathcal{O}\left(\mathbb{C} P^{1}\right)} \mathcal{O}(2) \\
& \longrightarrow H^{2}(B) \otimes_{\mathbb{C}} \mathcal{O}(2) \longrightarrow 0
\end{aligned}
$$

where $\hat{\mathcal{O}}_{s_{x_{i}}}$ is the $s_{x_{i}}$-adic completion of $\mathcal{O}_{\mathrm{Tw}(M)}$, and $s_{x_{i}} \subset \mathrm{Tw}(M)$ is the rational curve $x_{i} \times \mathbb{C} P^{1} \subset \mathrm{Tw}(M)$, and $\operatorname{Hom}_{\mathbb{C}}\left(\hat{\mathcal{O}}_{s_{x_{i}}}, \mathbb{C}\right)$ the sheaf of continuous (in adic

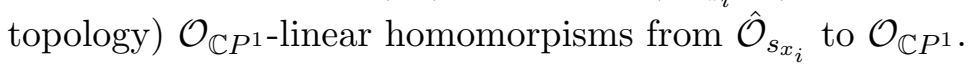

Proof: The exact sequence (7.12) is a relative version of (7.10). By Proposition 6.3 , we have $R^{i} \pi_{*} \operatorname{Tw}(B) \cong H^{i}(B) \otimes_{\mathbb{C}} \mathcal{O}(i)$; this gives us the rightmost and the 
leftmost term of the exact sequence (7.12). To prove (7.12) it remains to show that

$$
\begin{aligned}
& \bigoplus_{x_{i} \in A} \operatorname{Hom}_{\mathbb{C}}\left(\hat{\mathcal{O}}_{s_{x_{i}}}, \mathbb{C}\right) \otimes_{\mathcal{O}_{\mathrm{Tw}(M)}} \operatorname{Tw}(B) \otimes_{\mathcal{O}\left(\mathbb{C} P^{1}\right)} \mathcal{O}(2) \\
& \quad \cong \mathcal{H}_{A \times \mathbb{C} P^{1}}^{1}(\operatorname{Tw}(B))
\end{aligned}
$$

The fiberwise canonical class of $\operatorname{Tw}(M)$ is $\mathcal{O}(2)$ because the normal bundle to $s_{x_{i}}$ is $\mathcal{O}(1) \oplus \mathcal{O}(1)$. Therefore, (7.13) is implied by Proposition 7.5.

To prove that $H^{2}\left(\mathrm{Tw}_{A} B\right)=0$, notice that $R^{2} \pi_{*} \operatorname{Tw}_{A}(B)=0$ because the fibers of $\pi: \operatorname{Tw}(M \backslash A) \longrightarrow \mathbb{C} P^{1}$ have cohomological dimension $\leqslant 1$ (Corollary 7.6). Therefore,

$$
H^{2}\left(\operatorname{Tw}_{A}(B)\right)=H^{1}\left(R^{1} \pi_{*} \operatorname{Tw}_{A}(B)\right) .
$$

On the other hand, the sheaf $R^{1} \pi_{*} \mathrm{Tw}_{A}(B)$ can be obtained in an explicit way from the exact sequence (7.12) as follows.

Consider the $s_{x_{i}}$-adic filtration on $\hat{\mathcal{O}}_{s_{x_{i}}}$. The associated graded sheaf is isomorphic to $\oplus_{j} \mathfrak{m}_{s_{i}}^{j-1} / \mathfrak{m}_{s_{i}}^{j}$, where $\mathfrak{m}_{s_{i}}$ is the maximal ideal of $s_{x_{i}}$. However,

$$
\mathfrak{m}_{s_{i}}^{j-1} / \mathfrak{m}_{s_{i}}^{j} \cong \mathcal{O}(-j) \otimes_{\mathbb{C}} S^{j} \Lambda_{x_{i}}^{1,0}(M, I),
$$

because $\mathfrak{m}_{s_{i}}^{j-1} / \mathfrak{m}_{s_{i}}^{j}$ is the $j$-th symmetric power of

$$
\mathfrak{m}_{s_{i}} /\left.\mathfrak{m}_{s_{i}}^{2} \cong \Lambda_{\pi}^{1,0}(\operatorname{Tw}(M))\right|_{s_{x_{i}}} \cong N^{*}\left(s_{x_{i}}\right)
$$

and the normal bundle $N\left(s_{x_{i}}\right)$ is isomorphic to $\mathcal{O}(1)^{\oplus^{2}}$.

The $\mathfrak{m}_{s_{i}}$-adic filtration on $\hat{\mathcal{O}}_{s_{x_{i}}}$ induces a filtration on

$$
\operatorname{Hom}_{\mathbb{C}}\left(\hat{\mathcal{O}}_{s_{x_{i}}}, \mathbb{C}\right)
$$

with the associated graded quotient sheaves isomorphic to

$$
\mathcal{O}(j) \otimes_{\mathbb{C}} S^{j} T_{x_{i}}^{1,0}(M, I)
$$

These bundles are positive, for all $j$. This allows us to represent

$$
\operatorname{Hom}_{\mathbb{C}}\left(\hat{\mathcal{O}}_{s_{x_{i}}}, \mathbb{C}\right)
$$

as a direct limit of of positive bundles:

$$
\operatorname{Hom}_{\mathbb{C}}\left(\hat{\mathcal{O}}_{s_{x_{i}}}, \mathbb{C}\right)=\underline{\lim } \operatorname{Hom}_{\mathbb{C}}\left(\mathcal{O}_{s_{x_{i}}} / \mathfrak{m}_{s_{i}}^{j}, \mathbb{C}\right),
$$


with all $\operatorname{Hom}_{\mathbb{C}}\left(\mathcal{O}_{s_{x_{i}}} / \mathfrak{m}_{s_{i}}^{j}, \mathbb{C}\right)$ admitting a filtration with positive associated graded quotients, hence positive. We obtain that $\operatorname{Hom}_{\mathbb{C}}\left(\hat{\mathcal{O}}_{s_{x_{i}}}, \mathbb{C}\right)$ is a direct limit of positive bundles. From the exact sequence (7.12) we obtain

$$
\begin{aligned}
& 0 \longrightarrow H^{1}(B) \otimes_{\mathbb{C}} \mathcal{O}(1) \longrightarrow R^{1} \pi_{*} \operatorname{Tw}_{A}(B) \\
& \longrightarrow \oplus_{\alpha_{k}} \mathcal{O}\left(\alpha_{k}\right) \longrightarrow H^{2}(B) \otimes_{\mathbb{C}} \mathcal{O}(2) \longrightarrow 0, \\
& \quad \alpha_{k} \geqslant 2 .
\end{aligned}
$$

This implies immediately that $R^{1} \pi_{*} \operatorname{Tw}_{A}(B)$ is a direct sum of $\mathcal{O}(k)$ with $k \geqslant 1$, and its first cohomology vanish. Now (7.14) implies $H^{2}\left(\operatorname{Tw}_{A}(B)\right)=0$. We proved Proposition 7.9.

Let $M$ be a hyperkähler manifold, and $x \in M$ the point. By $s_{x} \subset \operatorname{Tw}(M)$ we denote the rational curve $\mathbb{C} P^{1} \times\{x\}$. Consider the local cohomology sheaf

$$
D_{s_{x}}:=\mathcal{H}_{s_{x}}^{\operatorname{dim}_{\mathbb{C}} M}\left(\mathcal{O}_{\mathrm{Tw}(M)}\right)=\operatorname{Hom}_{\mathbb{C}}\left(\hat{\mathcal{O}}_{s_{x}}, \mathbb{C}\right) \otimes_{\mathcal{O}_{\mathrm{Tw}(M)}} K_{\pi}(\mathrm{Tw}(M))
$$

(the last equality holds by Proposition 7.5). Using (7.4), we can trivialise the infinite-dimensional bundle $\hat{\mathcal{O}}_{s_{x}}$ outside of $I$ in a natural way. This gives a trivialization of $D_{s_{x}}$ outside of $I$ (the bundle $K_{\pi}(\operatorname{Tw}(M)) \cong \mathcal{O}\left(\operatorname{dim}_{\mathbb{C}} M\right)$ is trivialized by taking the sections which have a zero of order $n$ in $I)$. Given a hyperholomorphic bundle $B$ on $M$, we obtain a trivialization of $\operatorname{Tw}(B)$ in a neighbourhood of $s_{x} \backslash I \subset \operatorname{Tw}(M) \backslash(M, I)$ (see (7.5)). This gives a trivialization of the term

$$
\bigoplus_{x_{i} \in A} \operatorname{Hom}_{\mathbb{C}}\left(\hat{\mathcal{O}}_{s_{x_{i}}}, \mathbb{C}\right) \otimes_{\mathcal{O}_{\mathrm{Tw}(M)}} \operatorname{Tw}(B) \otimes_{\mathcal{O}\left(\mathbb{C} P^{1}\right)} \mathcal{O}(2)
$$

from the exact sequence (7.12). The sheaf $R^{2} \pi_{*} \operatorname{Tw}(B)=H^{2}(B) \otimes_{\mathbb{C}} \mathcal{O}(2)$ is trivialized outside $I$ by taking the sections with second order zeroes at $I$. We obtained a trivialization of the sheaf

$$
\begin{aligned}
& \operatorname{ker}\left(\bigoplus_{x_{i} \in A} \operatorname{Hom}_{\mathbb{C}}\left(\hat{\mathcal{O}}_{s_{x_{i}}}, \mathbb{C}\right) \otimes_{\mathcal{O}_{\operatorname{Tw}(M)}} \operatorname{Tw}(B) \otimes_{\mathcal{O}\left(\mathbb{C} P^{1}\right)} \mathcal{O}(2)\right. \\
& \left.\quad \longrightarrow R^{2} \pi_{*} \operatorname{Tw}(B)\right)\left.\right|_{\mathbb{C} P^{1} \backslash I} .
\end{aligned}
$$

Corollary 7.10: In the above assumptions, let $\nu$ be a section of the sheaf (7.16) which is compatible with the trivialization constructed above. Then $\nu$ van 
be extended to a holomorphic section of the sheaf

$$
\begin{aligned}
& \operatorname{ker}\left(\bigoplus_{x_{i} \in A} \operatorname{Hom}_{\mathbb{C}}\left(\hat{\mathcal{O}}_{s_{x_{i}}}, \mathbb{C}\right) \otimes_{\mathcal{O}_{\mathrm{Tw}(M)}} \operatorname{Tw}(B) \otimes_{\mathcal{O}\left(\mathbb{C} P^{1}\right)} \mathcal{O}(2)\right. \\
& \left.\quad \longrightarrow R^{2} \pi_{*} \operatorname{Tw}(B)\right) .
\end{aligned}
$$

on $\mathbb{C} P^{1}$.

Proof: Follows immediately from Proposition 7.2.

\section{Coherent sheaves on Generic COMPACt tori}

\subsection{Reflexive sheaves on generic complex tori are bundles. Theorem}

8.1: Let $T$ be a compact complex torus, $\operatorname{dim}_{\mathbb{C}} T>2$. Assume that $H^{p, p}(T) \cap$ $H^{2 p}(T, \mathbb{Z})=0$, for all $0<p<\operatorname{dim}_{\mathbb{C}} T{ }^{1}$ Then all coherent sheaves on $T$ have isolated singularities and smooth reflexive hulls.

Proof: Consider a coherent sheaf $F$ on $T$. Since $T$ has no non-trivial integer $(p, p)$-cycles, all subvarieties of $T$ are finite sets of points. Therefore, $F$ has isolated singularities. It remains to prove that the reflexive hull $E=F^{* *}$ is smooth. The following argument is well known (see e.g. [Voi]).

Lemma 8.2: Let $T$ be a compact complex torus with no non-trivial integer $(p, p)$-cycles, for $0<p<3$, and $E$ a reflexive coherent sheaf on $T$. Then $E$ is a bundle.

Proof: We may assume that $\operatorname{dim}_{\mathbb{C}} \geqslant 3$. Since $T$ has no non-trivial integer $(p, p)$-cycles, the first Chern class of all sheaves on $T$ vanishes. Therefore, all coherent sheaves on $T$ are semistable. Consider a filtration

$$
0=E_{0} \subset E_{1} \subset \ldots \subset E_{n}=E
$$

with all the subfactors $E_{i} / E_{i-1}$ stable. First of all, we show that the reflexizations of the sheaves $E_{i} / E_{i-1}$ are smooth, for all $i$. By construction, these sheaves are stable. Assume that $E$ is stable and reflexive.

In [BS], Bando and Siu construct a canonical Hermitian Yang-Mills connection $\nabla$ on the non-singular part of any stable reflexive sheaf on a compact Kähler manifold $X$, with $L^{2}$-integrable curvature $\Theta$. Such a connection (called admissible

\footnotetext{
${ }^{1}$ This is equivalent to $T$ being Mumford-Tate generic.
} 
Yang-Mills) is unique. In [BS] (see also [T]) it was proven that the $L^{1}$-integrable form $\operatorname{Tr}(\Theta \wedge \Theta)$ (considered as a current on the Kähler manifold $X$ ) is closed and represents the cohomology class

$$
\operatorname{Tr}[\Theta \wedge \Theta]=c_{2}(E)-\frac{r-1}{r} c_{1}^{2}(E)
$$

Consider the standard Hodge operator $\Lambda$ on differential forms (see Definition 3.4). Since $\nabla$ is Yang-Mills, we have

$$
\Lambda^{2}\left(\Theta^{2}\right) \geqslant 0
$$

and the equality is reached only if $\Theta=0 .{ }^{2}$ Comparing (8.1) and (8.2), and using $c_{1}(E), c_{2}(E)=0$, we obtain that $\Theta=0$, that is, $\nabla$ is flat. Since $E$ is non-singular in codimension $2, \nabla$ has no local monodromy. Therefore, $(E, \nabla)$ can be extended to a flat holomorphic bundle on $T$. By Lemma 5.7, this extension is a reflexization of $E$.

We proved that $E^{* *}$ is smooth, for all stable coherent sheaves $E$ on $T$. This implies that any vector bundle $E$ on $T$ is filtered by subsheaves $E_{i}$, with the quotient sheaves $E_{i} / E_{i-1}$ all having smooth reflexizations. Replacing $E_{i}$ by its reflexization $E_{i}^{* *} \subset E$, we may assume that al $E_{i}$ are reflexive. Using induction, we may assume also that $E_{n-1}$ is smooth.

We have an exact sequence

$$
0 \longrightarrow E_{n-1} \longrightarrow E \longrightarrow E_{n} / E_{n-1} \longrightarrow 0
$$

with $E_{n-1}$ smooth, $E$ reflexive, and $F=E_{n} / E_{n-1}$ a stable sheaf having (as we have shown above) a smooth reflexization.

Then $E$ is given by a class $\nu \in \operatorname{Ext}^{1}\left(F, E_{n-1}\right)$. Consider the exact sequence

$$
0 \longrightarrow F \longrightarrow F^{* *} \longrightarrow C \longrightarrow 0
$$

where $C$ is a torsion sheaf (cokernel of the reflexization map). This gives a long exact sequence

$$
\operatorname{Ext}^{1}\left(F^{* *}, E_{n-1}\right) \longrightarrow \operatorname{Ext}^{1}\left(F, E_{n-1}\right) \stackrel{\delta}{\longrightarrow} \operatorname{Ext}^{2}\left(C, E_{n-1}\right)
$$

The kernel of $\delta$ in (8.3) corresponds to all extensions $\gamma \in \operatorname{Ext}^{1}\left(F, E_{n-1}\right)$ with a reflexization isomorphic to an extension of $F^{* *}$ with $E_{n-1}$. Clearly, such extensions are reflexive only if $C=0$. To prove that $C=0$, it suffices to show that

\footnotetext{
${ }^{2}$ This is the celebrated Lübke inequality [Lü], which implies flatness of stable bundles with zero Chern classes and the Bogomolov-Miyaoka-Yau inequality; see [BS], [T]
} 
$\delta(\nu)=0$. However, $C$ is a torsion sheaf, therefore its support $S$ is a finite set. By Grothendieck's duality (Proposition 7.5), the group $\operatorname{Ext}^{2}\left(C, E_{n-1}\right) \subset H_{S}^{2}\left(E_{n-1}\right)$ vanishes, for $\operatorname{dim} T>2$. Therefore, $E$ is smooth. This proves Lemma 8.2.

The same argument also proves the following corollary.

Corollary 8.3: In assumptions of Lemma 8.2, let

$$
0 \longrightarrow F_{1} \longrightarrow F_{2} \longrightarrow F_{3} \longrightarrow 0
$$

be an exact sequence of coherent sheaves, with $F_{1}, F_{2}$ reflexive (hence, by Lemma 8.2 , locally free). Then the sheaf $F_{3}$ is also locally free.

Let $T$ be a compact complex torus of complex dimension $>2$ which has no non-trivial integer $(p, p)$-cycles, $0<p<3$. Since $c_{1}(B)=0$ for any sheaf $B$ on $T$, any sheaf on $T$ is semistable. Consider a Jordan-Hölder filtration, with polystable associate quotient sheaves $B_{i}$. By Lemma 8.2, the reflexization $B_{i}^{* *}$ is a bundle. However, the sheaves $B_{i}$ are not necessarily bundles. Indeed, let $X$ be a complex manifold and consider an extension

$$
0 \longrightarrow \mathcal{O}_{\Omega} \longrightarrow B \longrightarrow I_{x} \longrightarrow 0
$$

where $I_{x} \subset \mathcal{O}_{X}$ is the ideal sheaf of a point $x$. If the extension (8.4) is non-trivial, and $X$ is Stein, then $B$ is a bundle, as an easy check shows. It is easy to construct such an extension for $n=2$. However, over a generic complex torus of dimension $>2$ this situation is impossible, as the following trivial proposition implies.

Proposition 8.4: Let $T$ be a $d$-dimensional compact complex torus, $d \geqslant 3$. Assume that $T$ has no non-trivial integer $(p, p)$-cycles:

$$
H^{p, p}(T) \cap H^{2 p}(T, \mathbb{Z})=0, \text { for } 0<p<d .
$$

Then any holomorphic vector bundle on $T$ admits a locally free filtration by holomorphic bundles with associated graded sheaves locally free of rank 1 .

Proof: Consider a Jordan-Hölder filtration

$$
0=F_{0} \subset F_{1} \subset \ldots \subset F_{n}=B,
$$

with associated graded sheaves stable. If $F_{i}$ are not reflexive, we replace $F_{i}$ by $F_{i}^{* *} \subset B$. Therefore, we may assume that the sheaves $F_{i}$ are reflexive. Corollary 8.3 implies that for all $i$, the quotient $F_{i} / F_{i-1}$ is a bundle. These bundles are also stable, and therefore admit a Yang-Mills metrics. By Lübke's (also Bogomolov's and Simpson's) argument ([S]) a Yang-Mills connection on a bundle with the 
zero Chern classes $c_{1}, c_{2}$ is flat. Thus, it corresponds to an irreducible unitary representation of $\pi_{1}(T)$. However, $\pi_{1}(T)$ is abelian, hence all its irreducible unitary representations are 1-dimensional. We proved that $F_{i} / F_{i-1}$ is a line bundle. Proposition 8.4 is proven.

Consider a holomorphic vector bundle $B$ on a compact complex $d$-dimensional torus $T$ with

$$
H^{p, p}(T) \cap H^{2 p}(T, \mathbb{Z})=0, \text { for } 0<p<d .
$$

In this Subsection we prove that $B$ admits a natural flat connection $\nabla$ compatible with a holomorphic structure. If $\nabla$ is flat and Hermitian, then it is Yang-Mills. In this case, $B$ is polystable by Uhlenbeck-Yau theorem (Theorem 3.5).

Generally speaking, $B$ is not stable, and $\nabla$ is not Hermitian.

Let $B_{1}, B_{2}$ be flat holomorphic Hermitian vector bundles on $T$. Using the Hodge theory, we identify $\operatorname{Ext}^{1}\left(B_{1}, B_{2}\right)$ with the space of harmonic $(0,1)$-forms with coefficients in $\operatorname{Hom}\left(B_{1}, B_{2}\right)$. The maximum principle implies that any harmonic $\operatorname{Hom}\left(B_{1}, B_{2}\right)$-valued form is parallel with respect to the natural flat connection on $\Lambda^{1}(M) \otimes B_{1}^{*} \otimes B_{2}$. Let $B$ be the Yoneda extension of $B_{2}$ with $B_{1}$ :

$$
0 \longrightarrow B_{1} \longrightarrow B \longrightarrow B_{2} \longrightarrow 0 \text {. }
$$

The holomorphic structure operator in $B$ can be written explicitly as follows. Let $\nu \in \mathcal{H}^{0,1}\left(\operatorname{Hom}\left(B_{2}, B_{1}\right)\right.$ be the harmonic representative of the extension class defining (8.5), and $\bar{\partial}_{1}, \bar{\partial}_{2}$ the holomorphic structure operators on $B_{1}, B_{2}$,

$$
\bar{\partial}_{i}: B_{i} \longrightarrow B_{i} \otimes \Lambda^{0,1}(M) .
$$

Consider the holomorphic structure operator $\bar{\partial}_{g r}:=\bar{\partial}_{1}+\bar{\partial}_{2}$ on $B_{g r}:=B_{1} \oplus B_{2}$. We define an operator

$$
\bar{\partial}: B_{g r} \longrightarrow B_{g r} \otimes \Lambda^{0,1}(M)
$$

as

$$
\bar{\partial}:=\bar{\partial}_{g r}+\nu
$$

where

$$
\nu \in \mathcal{H}^{0,1}\left(\operatorname{Hom}\left(B_{2}, B_{1}\right)\right) \subset \Lambda^{0,1}\left(\operatorname{End}\left(B_{g r}\right)\right)
$$

is understood as a connection form. We have

$$
\bar{\partial}^{2}=\bar{\partial}_{g r}(\nu)+2 \nu \wedge \nu=0
$$


(this is the famous Maurer-Cartan equation). ${ }^{3}$. Therefore, $\bar{\partial}$ is a holomorphic structure operator. Clearly, the holomorphic bundle $\left(B_{1} \oplus B_{2}, \bar{\partial}\right)$ is isomorphic to $B$.

Given an arbitrary harmonic form $\nu \in \mathcal{H}^{0,1}\left(\operatorname{End}\left(B_{g r}\right)\right)$, the Maurer-Cartan equation (8.6) will not, generally speaking, hold. The main purpose of deformation theory is to find its solutions in terms of the cohomology classes.

However, over a torus we may use the flatness of harmonic representatives to obtain the solutions of (8.6) in a straightforward way. The following theorem is the main result of this Subsection.

Theorem 8.5: Let $T$ be a compact complex torus, $B_{1}, \ldots B_{n}$ flat holomorphic Hermitian vector bundles and $B$ a holomorphic vector bundle with a filtration

$$
0=E_{0} \subset E_{1} \subset \ldots \subset E_{n}=B,
$$

such that $E_{i} / E_{i-1} \cong B_{i}$. Then the following assertions are true.

(i): The holomorphic structure on $B$ can be obtained as follows. Identify $B$ with $B_{g r}:=\oplus B_{i}$ as $C^{\infty}$-bundle. Then there is a cohomology class $\nu$

$$
\nu \in \bigoplus_{i>j} \operatorname{Ext}^{1}\left(B_{i}, B_{j}\right) \subset \operatorname{Ext}^{1}\left(B_{g r}, B_{g r}\right)
$$

such that the holomorphic structure operator in $B$ is written as

$$
\bar{\partial}=\bar{\partial}_{g r}+\nu_{0},
$$

where $\bar{\partial}_{g r}$ is the holomorphic structure operator on $B_{g r}$, and

$$
\nu_{0} \in \Lambda^{0,1}\left(\operatorname{End}\left(B_{g r}\right)\right)
$$

denotes the harmonic representative of $\nu$.

(ii): The class $\nu \in \oplus_{i>j} \operatorname{Ext}^{1}\left(B_{i}, B_{j}\right)$ is unique up to an automorphism of the bundle $B_{g r}=\oplus B_{i}$ preserving the filtration

$$
B_{1} \subset B_{1} \oplus B_{2} \subset \ldots \subset \oplus_{i \leqslant k} B_{i} \subset \ldots
$$

(iii): Given an arbitrary cohomology class

$$
\nu \in \bigoplus_{i>j} \operatorname{Ext}^{1}\left(B_{i}, B_{j}\right)
$$

\footnotetext{
${ }^{3}$ The quadratic term $\nu \wedge \nu$ vanishes in our case automatically. This follows because $\nu \wedge \nu$ is $\bar{\partial}_{g r}$-harmonic and $\bar{\partial}_{g r}$-exact
} 
with $\nu^{2}=0$, we can reconstruct the holomorphic bundle $B$ as follows: $B$ is identified with $B_{g r}$ as a $C^{\infty}$-bundle, and the holomorphic structure operator in $B$ is defined as in (8.8).

Remark 8.6: By Proposition 8.4, on a complex compact torus $T$ of complex dimension $d>2$ with

$$
H^{p, p}(T) \cap H^{2 p}(T, \mathbb{Z})=0, \text { for } 0<p<3,
$$

every vector bundle can be obtained this way. Moreover, in this situation, the bundles $B_{i}$ can be chosen 1-dimensional.

Proof of Theorem 8.5: Write the holomorphic structure operator on $B$ as $\bar{\partial}=\bar{\partial}_{g r}+\widetilde{\nu}$, where $\widetilde{\nu}$ is a $(0,1)$-form with values in

$$
\oplus_{i>j} \Lambda^{0,1}\left(T, \operatorname{Hom}\left(B_{i}, B_{j}\right)\right) .
$$

The form $\widetilde{\nu}$ satisfies the Maurer-Cartan equation (8.6). However, this form is, generally speaking, not harmonic. Every automorphism $g \in$ End $B_{g r}$ acts on $\widetilde{\nu}$ as $\widetilde{\nu} \longrightarrow g(\widetilde{\nu})+\bar{\partial}_{g r}(g)$ (this is the well-known gauge action). To produce $\nu$ with the properties described in Theorem 8.5 (i), we need to find a correct gauge transform.

Consider the group $\left(\mathbb{C}^{*}\right)^{n}$ acting on $B_{g r}$ by diagonal automorphisms, in such a way that the $i$-th component $\alpha_{i}$ of $\left(\mathbb{C}^{*}\right)^{n}$ acts trivially on $B_{j} \subset B_{g r}$ for $i \neq j$, and as a multiplication by $\alpha_{i}$ on $B_{i}$.

We shall write the action of $\left(\mathbb{C}^{*}\right)^{n}$ on $\Lambda^{0,1}\left(\operatorname{End}\left(B_{g r}\right)\right.$ as follows. Let

$$
\begin{aligned}
& \widetilde{\nu} \in \Lambda^{0,1}\left(\operatorname{End}\left(B_{g r}\right)=\oplus_{i, j} \Lambda^{0,1}\left(B_{i}, B_{j}\right),\right. \\
& \widetilde{\nu}:=\sum_{i, j} \widetilde{\nu}_{i j}, \quad \widetilde{\nu}_{i j} \in \Lambda^{0,1}\left(B_{i}, B_{j}\right)
\end{aligned}
$$

If $\alpha \in\left(\mathbb{C}^{*}\right)^{n}, \alpha=\prod_{i} \alpha_{i}$, then

$$
\alpha(\widetilde{\nu})=\sum_{i, j} \alpha_{i} \alpha_{j}^{-1} \widetilde{\nu}_{i j}
$$

The group $\left(\mathbb{C}^{*}\right)^{n}$ acts in this fashion on the solutions of Maurer-Cartan equation, and maps every solution to an equivalent one. If $\alpha_{j} \gg \alpha_{i}$ for all $i>j$, then $\alpha$ maps

$$
\widetilde{\nu} \in \oplus_{i>j} \Lambda^{0,1}\left(T, \operatorname{Hom}\left(B_{i}, B_{j}\right)\right)
$$

to a form which is arbitrarily small. 
Consider the local deformation space $\operatorname{Def}\left(B_{g r}\right)$ for $B_{g r}$, constructed in [ST]. The above argument implies that every neighbourhood of the point $\left[B_{g r}\right] \in$ $\operatorname{Def}\left(B_{g r}\right)$ contains a bundle which is isomorphic to $B$.

Let $E$ be a holomorphic vector bundle over a compact Kähler manifold. The local deformation space $\operatorname{Def}(E)$ can be constructed explicitly in terms of Massey products as follows.

One can define the Massey products as obstructions to constructing a solution of the Maurer-Cartan equation (see e.g. [BT], or [May], [Re] for a more classical approach). Locally, $\operatorname{Def}(E)$ is embedded to the vector space $\operatorname{Ext}^{1}(E, E)$, and the image of this embedding is a germ of all vectors $\theta \in \operatorname{Ext}^{1}(E, E)$, such that $\theta \wedge \theta=0$ and all the higher Massey products of $\theta$ with itself vanish.

Fix such a vector $\theta \in \operatorname{Ext}^{1}(E, E)$. We construct the corresponding vector bundle $E_{\theta} \in \operatorname{Def}(E)$ using the Hodge theory as follows (see e.g. [V0]).

Let $\theta_{0} \in \mathcal{H}^{0,1}(\operatorname{Hom}(E, E))$ be the harmonic representative of $\theta$. Using induction, we define

$$
\theta_{n}:=-\frac{1}{2} G_{\bar{\partial}} \sum_{i+j=n-1} \theta_{i} \wedge \theta_{j}
$$

where $G_{\bar{\partial}}$ is the Green operator inverting the holomorphic structure operator

$$
\bar{\partial}: \Lambda^{0, k} \otimes E \longrightarrow \Lambda^{0, k+1} \otimes E .
$$

on its image. The Green operator $G_{\bar{\partial}}$ is compact. This can be used to show that for $\theta$ sufficiently small, the series $\widetilde{\theta}:=\sum \theta_{i}$ converges. The vanishing of Massey products is equivalent to the following condition

$$
\bar{\partial} \theta_{n}=-\frac{1}{2} \sum_{i+j=n-1} \theta_{i} \wedge \theta_{j}
$$

which is apparent from the definition given in $[\mathrm{BT}]$. In this case, we have

$$
\bar{\partial} \widetilde{\theta}=-\frac{1}{2} \widetilde{\theta} \wedge \widetilde{\theta}
$$

and $\widetilde{\theta}$ is a solution of the Maurer-Cartan equation (8.6). Therefore, the operator $\bar{\partial}_{\theta}=\bar{\partial}+\widetilde{\theta}$ satisfies $\bar{\partial}_{\theta}^{2}=0$, and by Newlander-Nirenberg theorem (Theorem 3.1) this operator defines a holomorphic structure on $E$. On the deformation space $\operatorname{Def}(E) \subset \operatorname{Ext}^{1}(E, E)$, the point $\theta$ corresponds to a bundle $\left(E, \bar{\partial}_{\theta}\right)$. 
Now we return to holomorphic bundles over a compact torus and the proof of Theorem 8.5. We obtain that $B$ is given by some $\nu \in \operatorname{Ext}^{1}\left(B_{g r}, B_{g r}\right)$. Since $B$ and $\oplus B_{i}$ have compatible filtrations, by functoriality we may assume that

$$
\nu \in \oplus_{i>j} \operatorname{Ext}^{1}\left(B_{i}, B_{j}\right) .
$$

The higher Massey operations in $\operatorname{Ext}^{*}\left(B_{g r}, B_{g r}\right)$ vanish, because the bundle $B_{g r}$ is flat (the same proof works as was used in [DGMS]; see also [GM]). Therefore, $B$ can be reconstructed from $\nu$ for any $\nu$ such that the cohomology class $\nu \wedge \nu$ vanishes. Pick a harmonic representative $\nu_{0}$ of $\nu$. Since $B_{g r}$ is flat, $\nu_{0}$ is parallel. Therefore $\nu_{0} \wedge \nu_{0}$ is also parallel, hence harmonic. We obtain that the cohomology class of $\nu_{0} \wedge \nu_{0}$ vanishes if and only if this form vanishes identically.

Starting from a bundle $B$ with a filtration satisfying the assumptions of Theorem 8.5, we have constructed a cohomology class $\nu \in \oplus_{i>j} \operatorname{Ext}^{1}\left(B_{i}, B_{j}\right)$, with $\nu \wedge \nu=0$. The harmonic representative $\nu_{0}$ of $\nu$ satisfies $\nu_{0} \wedge \nu_{0}=0$. Therefore, $\nu_{0}$ is a solution of Maurer-Cartan equation, and $\bar{\partial}_{g r}+\nu_{0}$ is equivalent to the holomorphic structure operator of $B$. This proves Theorem 8.5 (i). Theorem 8.5 (ii) is clear by functoriality of our construction, and Theorem 8.5 (iii) is obvious.

We also obtained the following corollary.

Corollary 8.7: Let $B$ be a holomorphic vector bundle on a compact torus $T$, $\operatorname{dim}_{\mathbb{C}} T>2$,

$$
H^{p, p}(T) \cap H^{2 p}(T, \mathbb{Z})=0, \text { for } 0<p<3
$$

Then $B$ admits a flat connection compatible with the holomorphic structure.

Proof: By Proposition 8.4, $B$ admits a filtration satisfying the assumptions of Theorem 8.5. Let $\nu_{0} \in \Lambda^{0,1}\left(\operatorname{End}\left(B_{g r}\right)\right)$ be the $(0,1)$-form which defines the holomorphic structure on $B$ as in (8.8):

$$
\bar{\partial}=\bar{\partial}_{g r}+\nu_{0}
$$

and let $\nabla_{g r}$ be the standard flat Hermitian connection on $B_{g r}$. Then the connection $\nabla:=\nabla_{g r}+\nu_{0}$ is flat. By construction, the $(0,1)$-part of $\nabla$ is equal to the holomorphic structure operator on $B$.

The form $\nu_{0}$ is called the Higgs field of the holomorphic bundle $B$. 


\section{YonedA EXTENSION IN ABELIAN CATEGORIES}

In this Section we study abelian categories of finite length, in quite an abstract setting. Further on, these results are applied to the category of reflexive sheaves on a hyperkähler manifold.

\subsection{Abelian categories of cohomological dimension $\leqslant 1$. Definition 9.1:}

Let $\mathcal{C}$ be an abelian category. An object $B \in \mathcal{C}$ is called simple if it has no proper sub-objects: for all $B^{\prime} \subset B$, either $B^{\prime}=B$ or $B^{\prime}=0$. An object $B$ is called semisimple if $B$ is a direct sum of simple objects.

An abelian category $\mathcal{C}$ is called of finite length if every object $B \in \mathcal{C}$ is a finite extension of simple objects. Equivalently, $\mathcal{C}$ is of finite length if any increasing or decreasing chain of sub-objects of $B$ stabilizes, for all $B \in \mathcal{C}$. $^{1}$

Let $\mathcal{C}$ be an abelian category. Denote by $\operatorname{Ext}^{1}\left(B, B^{\prime}\right)$ the group of Yoneda extensions from $B$ to $B^{\prime}$. Given an exact sequence

$$
0 \longrightarrow B_{1} \longrightarrow B_{2} \longrightarrow B_{3} \longrightarrow 0
$$

we have the exact sequences

$$
\begin{aligned}
0 \longrightarrow \operatorname{Hom}\left(B_{3}, B\right) \longrightarrow \operatorname{Hom}\left(B_{2}, B\right) \longrightarrow \operatorname{Hom}\left(B_{1}, B\right) \longrightarrow \\
\longrightarrow \operatorname{Ext}^{1}\left(B_{3}, B\right) \longrightarrow \operatorname{Ext}^{1}\left(B_{2}, B\right) \longrightarrow \operatorname{Ext}^{1}\left(B_{1}, B\right)
\end{aligned}
$$

and

$$
\begin{aligned}
0 \longrightarrow \operatorname{Hom}\left(B, B_{1}\right) \longrightarrow \operatorname{Hom}\left(B, B_{2}\right) \longrightarrow \operatorname{Hom}\left(B, B_{3}\right) \longrightarrow \\
\longrightarrow \operatorname{Ext}^{1}\left(B, B_{1}\right) \longrightarrow \operatorname{Ext}^{1}\left(B, B_{2}\right) \longrightarrow \operatorname{Ext}^{1}\left(B, B_{3}\right) .
\end{aligned}
$$

The following elementary claim is well known

Claim 9.2: Let $\mathcal{C}$ be an abelian category. Then the following conditions are equivalent:

(i): The exact sequences (9.1), (9.2) are right exact

(ii): The second Yoneda extension group vanishes identically

(iii): Any exact sequence of length 4 splits onto a direct sum of two exact sequences of length 3 .

\footnotetext{
${ }^{1}$ One also says $\mathcal{C}$ satisfies the ascending and descending chain condition.
} 
Definition 9.3: Let $\mathcal{C}$ be an abelian category satisfying the conditions (i)-(iii) of Claim 9.2. We say that $\mathcal{C}$ has cohomological dimension $\leqslant 1$.

The following lemma is quite easy.

Lemma 9.4: Let $\mathcal{C}$ be an abelian category of finite length. Assume that the exact sequences $(9.1),(9.2)$ are right exact for all semisimple $B_{1}, B_{3}$. Then $\mathcal{C}$ is of cohomological dimension $\leqslant 1$.

Proof: Define the length of an object $F \in \mathbb{C}$ as a length of a minimal filtration on $B$ with semisimple associated graded factors. We denote the length of $F$ by $l(F)$.

We prove Lemma 9.4 by induction by $l(B), l\left(B_{i}\right)$. By assumptions of Lemma 9.4, the second Yoneda extension $\operatorname{Ext}^{2}\left(B, B^{\prime}\right)$ vanishes for all semisimple $B, B^{\prime}$. We need to show that $\operatorname{Ext}^{2}\left(B, B^{\prime}\right)=0$ for arbitrary $B, B^{\prime}$. Using induction, we may assume that this group vanishes for all $B, B^{\prime}$ with $l(B)<n, l\left(B^{\prime}\right)<m$. Write an exact sequence

$$
0 \longrightarrow B^{\prime \prime} \longrightarrow B^{\prime} \longrightarrow B^{\prime \prime \prime} \longrightarrow 0
$$

with $l\left(B^{\prime \prime}\right), l\left(B^{\prime \prime \prime}\right)<l\left(B^{\prime}\right)$. From the long exact sequence

$$
\ldots \longrightarrow \operatorname{Ext}^{2}\left(B, B^{\prime \prime}\right) \longrightarrow \operatorname{Ext}^{2}\left(B, B^{\prime}\right) \longrightarrow \operatorname{Ext}^{2}\left(B, B^{\prime \prime \prime}\right) \longrightarrow \ldots
$$

and the induction assumption we obtain that $\operatorname{Ext}^{2}\left(B, B^{\prime}\right)=0$.

Lemma 9.5: Let $\mathcal{C} \subset \mathcal{C}_{0}$ be a full abelian subcategory of an abelian category $\mathcal{C}_{0}$. Assume that $\mathcal{C}_{0}$ is of cohomological dimension $\leqslant 2$. Then $\mathcal{C}$ is of cohomological dimension $\leqslant 1$.

Proof: Take a 4-term exact sequence in $\mathcal{C}$

$$
0 \longrightarrow F_{1} \longrightarrow F_{2} \longrightarrow F_{3} \longrightarrow F_{4} \longrightarrow 0 .
$$

Since $\mathcal{C}_{0}$ has cohomological dimension $\leqslant 1,(9.3)$ splits in $\mathcal{C}_{0}$ onto a direct sum of two 3 -term exact sequences. Since $\mathcal{C}$ is full in $\mathcal{C}_{0}$, all direct summands of objects in $\mathcal{C}$ also belong in $\mathcal{C}$. Therefore, these 3 -term exact sequences belong in $\mathcal{C}$, and (9.3) splits in $\mathcal{C}$ onto a direct sum of two 3 -term exact sequences.

9.2. Functors on abelian categories of finite length. The main result of this Section is the following elementary proposition 
Proposition 9.6: Let $\mathcal{C} \stackrel{\gamma}{\longrightarrow} \mathcal{C}^{\prime}$ be a functor of abelian categories of finite length satisfying the following properties

(i): $\gamma$ induces an equivalence on the categories of semisimple objects in $\mathcal{C}$, $\mathcal{C}^{\prime}$

(ii): $\gamma$ induces an equivalence on the Yoneda Ext ${ }^{1}$-groups

$$
\operatorname{Ext}^{1}\left(B_{1}, B_{2}\right) \longrightarrow \operatorname{Ext}^{1}\left(\gamma\left(B_{1}\right), \gamma\left(B_{2}\right)\right),
$$

where $B_{1}, B_{2}$ are semisimple objects in $\mathcal{C}$.

(iii): The categories $\mathcal{C}, \mathcal{C}^{\prime}$ are of cohomological dimension $\leqslant 1$.

Then $\gamma$ is an equivalence.

Proof: ${ }^{2}$ The proof is obtained via the trivial diagram-chasing argument. As in the proof of Lemma 9.4, we use an induction by the length of $B$. Let $B, B^{\prime} \in \mathcal{C}$ be objects of length $n$. Consider an exact sequence

$$
0 \longrightarrow B_{0} \longrightarrow B \longrightarrow B_{1} \longrightarrow 0
$$

with $l\left(B_{1}\right)=n-1$ and $B_{0}$ semisimple. There is a long exact sequence

$$
0 \rightarrow \operatorname{Hom}\left(B^{\prime}, B_{0}\right) \rightarrow \operatorname{Hom}\left(B^{\prime}, B\right) \rightarrow \operatorname{Hom}\left(B^{\prime}, B_{1}\right) \rightarrow \operatorname{Ext}^{1}\left(B^{\prime}, B_{0}\right) \rightarrow \ldots
$$

Applying $\gamma$, we obtain a commutative diagram with exact rows

$(9.4)$

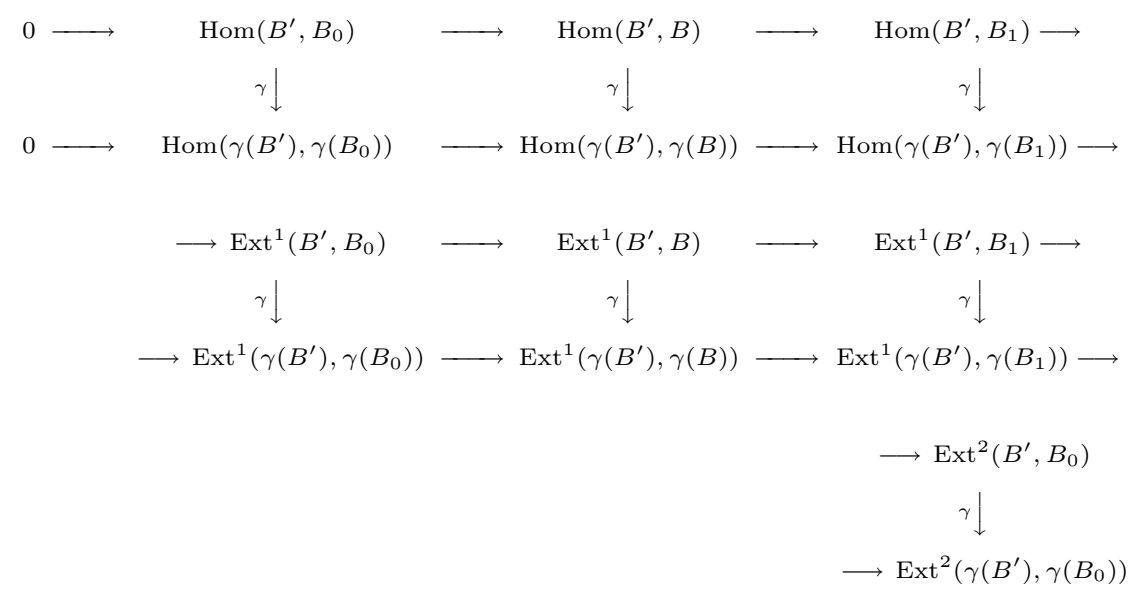

Since $\mathcal{C}, \mathcal{C}^{\prime}$ is of cohomological dimension $\leqslant 1$, we have

$$
\operatorname{Ext}^{2}\left(B^{\prime}, B_{0}\right)=\operatorname{Ext}^{2}\left(\gamma\left(B^{\prime}\right), \gamma\left(B_{0}\right)\right)=0 .
$$

\footnotetext{
${ }^{2}$ This proof was suggested by D. Kaledin.
} 
Using induction, we may assume that all vertical arrows of (9.4) are isomorphisms, except, possibly, the maps

$$
\operatorname{Ext}^{1}\left(B^{\prime}, B\right) \stackrel{\gamma}{\longrightarrow} \operatorname{Ext}^{1}\left(\gamma\left(B^{\prime}\right), \gamma(B)\right)
$$

and

$$
\operatorname{Hom}\left(B^{\prime}, B\right) \stackrel{\gamma}{\longrightarrow} \operatorname{Hom}\left(\gamma\left(B^{\prime}\right), \gamma(B)\right) .
$$

The snake lemma implies immediately that these two maps are isomorphisms as well. We proved that $\gamma$ is full and faithful and induces an isomorphism on Ext $^{1}$-groups. It remains to show that any object of $\mathcal{C}^{\prime}$ is isomorphic to $\gamma(B)$ for some $B \in \mathcal{C}$.

Consider an exact sequence

$$
0 \longrightarrow B_{0} \longrightarrow B \longrightarrow B_{1} \longrightarrow 0
$$

with $l\left(B_{1}\right)=n-1$ and $B_{0}$ semisimple. Using induction, we may assume that every object of length $\leqslant n-1$ is isomorphic to an object from the image of $\gamma$. Then, $B_{0}$ and $B_{1}$ belong to the image of $\gamma$. Since $B$ is represented by $\operatorname{Ext}^{1}\left(B_{1}, B_{0}\right)$, and $\gamma$ induces isomorphism on $\mathrm{Ext}^{1}$-groups, $B$ also belongs to the image of $\gamma$. This proves Proposition 9.6.

The same arguments as prove Proposition 9.6 can also be used to prove the following corollary

Corollary 9.7: Let $\mathcal{C} \stackrel{\gamma}{\longrightarrow} \mathcal{C}^{\prime}$ be a functor of abelian categories of finite length. Assume that $\gamma$ induces isomorphism on the respective subcategories of semisimple objects. Assume, moreover, that $\gamma$ induces a monomorphism on the Yoneda Ext ${ }^{1}$-groups between semisimple objects. Then $\gamma$ is full and faithful.

\section{Reflexive SHEAVES ON TWISTOR SPACES}

The aim of this section is the following theorem.

Theorem 10.1: Let $M$ be a hyperkähler K3 surface, or a hyperkähler compact torus. Assume that $M$ is generic in the sense of having no non-trivial $S U(2)$ invariant integer cycles:

$$
\left(H^{p}(M)_{S U(2)-i n v}\right) \cap H^{p}(M, \mathbb{Z})=0, \quad \text { for all } \quad 0<p<\operatorname{dim}_{\mathbb{R}} M .
$$


Consider generic ${ }^{1}$ complex structures $L, L^{\prime}$ induced by the hyperkähler structure, and let $\mathcal{C}^{r}(M, L), \mathcal{C}^{r}\left(M, L^{\prime}\right)$ be the categories of reflexive sheaves on $(M, L)$ and $\left(M, L^{\prime}\right)$. Then the categories $\mathcal{C}^{r}(M, L), \mathcal{C}^{r}\left(M, L^{\prime}\right)$ are equivalent. Moreover, this equivalence can be chosen in a canonical way if we chose another induced complex structure $I, I \neq L, L^{\prime}$.

Theorem 10.1 is proven in Subsection 10.3 for $M$ a surface, and in Subsection 10.2 for $M$ a compact torus, $\operatorname{dim}_{\mathbb{C}} M>2$.

10.1. Category $\mathcal{C}_{I}^{t w}$ : the definition. We work in assumptions of Theorem 10.1. Consider $(M, L)$ as a submanifold in $\operatorname{Tw}(M),(M, L)=\pi^{-1}(L)$. Consider the category

$$
\mathcal{C}^{r}:=\lim _{\longrightarrow} \mathcal{C}^{b}(\mathrm{Tw}(M \backslash S))
$$

where $\mathcal{C}^{b}(\operatorname{Tw}(M \backslash S))$ is the category of vector bundles on $\operatorname{Tw}(M \backslash S)$ which can be extended to $\operatorname{Tw}(M)$, and the limit is taken over all increasing sequences of finite subsets $S \subset M$. By Lemma 5.3, we may think of the objects of $\mathcal{C}^{r}$ as of reflexive sheaves on $\operatorname{Tw}(M)$; the space of morphisms of objects of $\mathcal{C}^{r}$ is isomorphic to the space of morphisms of the corresponding reflexive sheaves.

We are going to embed the category of reflexive sheaves on $(M, L)$ into the category $\mathcal{C}^{r}$ in such a way that the image is independent from the choice of $L$. Varying $L$, we obtain identifications between $\mathcal{C}^{r}(M, L)$ for various generic induced complex structures $L$. From the definition of $\mathcal{C}^{r}$ it follows that the natural restriction functor $R_{L}(\cdot):=\left.(\cdot)\right|_{(M, L)}$ maps $\mathcal{C}^{r}$ to the category $\mathcal{C}^{r}(M, L)$ of reflexive sheaves on $(M, L)$.

Fix an induced complex structure $I \neq L$ on $M$. We define the subcategory $\mathcal{C}_{I}^{t w} \subset \mathcal{C}^{r}$, in such a way that the restriction functor

$$
R_{L}: \mathcal{C}_{I}^{t w} \longrightarrow \mathcal{C}^{r}(M, L)
$$

is an equivalence, for all generic induced complex structures $L \neq I$.

In $[\mathrm{LY}]$, a theory of stable sheaves was constructed for some non-Kähler manifolds. In $[\mathrm{KV}]$, we proved that this theory can be applied to the twistor spaces. In particular, one may speak of semistable sheaves on $\operatorname{Tw}(M)$ and of Jordan-Hölder

\footnotetext{
${ }^{1}$ In the sense of Definition 2.8
} 
filtration, that is, a filtration with stable associated graded factors. As it happens in the usual case, the associated graded sheaf of a Jordan-Hölder filtration is independent from the choice of such filtration.

Definition 10.2: Let $M$ be a compact hyperkähler torus or a K3 surface, which is generic in the sense of having no non-trivial $S U(2)$-invariant integer cycles:

$$
\left(H^{p}(M)_{S U(2)-i n v}\right) \cap H^{p}(M, \mathbb{Z})=0, \quad \text { for all } \quad 0<p<\operatorname{dim}_{\mathbb{R}} M .
$$

The category $\mathcal{C}_{I}^{t w}$ is a full subcategory of the category of $\mathcal{C}^{r}$ reflexive sheaves on $\operatorname{Tw}(M)$, defined as follows. Consider a reflexive sheaf $F \in \mathcal{C}^{r}$. Then $F$ belongs to $\mathcal{C}_{I}^{t w}$ iff the following conditions hold

(i): The sheaf $F$ is semistable. The restriction of $F$ to $(M, I) \subset \operatorname{Tw}(M)$ has a polystable reflexization:

$$
\left(\left.F\right|_{(M, I)}\right)^{* *} \cong \oplus B_{i}
$$

where $B_{i}$ are hyperholomorphic bundles on $(M, I)$

(ii): There is a finite set $A \subset M$ such that $\left.\mathcal{F}\right|_{\operatorname{Tw}(M) \backslash(A \times\{I\})}$ is a bundle (that is, all singularities of $F$ are sitting in the finite set $A \times\{I\} \subset(M, I) \subset$ $\operatorname{Tw}(M))$.

(iii): Consider a Jordan-Hölder filtration of $F$

$$
0=F_{0} \subset F_{1} \subset \ldots \subset F_{n}=F
$$

with all $F_{i}$ reflexive, and the quotient sheaves $F_{i} / F_{i-1}$ stable. Then the quotient sheaves $F_{i} / F_{i-1}$ are non-singular on $\operatorname{Tw}(M \backslash A)$. For all $i$, the reflexization of $F_{i} / F_{i-1}$ is isomorphic to $\operatorname{Tw}\left(B_{i}\right)$, where $\operatorname{Tw}\left(B_{i}\right)$ is the twistor transform of the bundle $B_{i}$ considered in (i).

(iv): Let $x \in A$, and let $\operatorname{Tw}(M)_{x, I}$ be the germ of a neighbourhoof of $s_{x} \backslash I$ within $\operatorname{Tw}(M) \backslash(M, I)$ (see subsection 7.1). Then the sheaf $\left.F\right|_{\operatorname{Tw}(M)_{x, I}}$ can be trivialized over $s_{x} \backslash I$ by the map

$$
\tau:\left.F\right|_{\operatorname{Tw}(M)_{x_{0}, I}} \longrightarrow \xi^{*} F_{S} .
$$

(see (7.5) for details and notation). Moreover, this trivialization induces a trivialization on all subsheaves $F^{\prime} \subset F$. Whenether the quotient of 
two subsheaves $F_{1} / F_{2}$ has a reflexization isomorphic to $\operatorname{Tw}(B)$, then $\tau$ induces the same trivialization as (7.5) gives.

We consider objects of $\mathcal{C}_{I}^{t w}$ as reflexive sheaves on $\operatorname{Tw}(M)$.

Lemma 10.3: The category $\mathcal{C}_{I}^{t w}$ is abelian.

Proof: A full additive subcategory of an abelian category is abelian if and only if for any morphism $\varphi$ of this subcategory, the kernel and the cokernel of $\varphi$ belongs to this subcategory. Let $F, F^{\prime} \in \mathcal{C}_{I}^{t w}$, and let $\varphi: F \longrightarrow F^{\prime}$ be any morphism. Since $F, F^{\prime}$ is semistable, the sheaves $\operatorname{ker} \varphi$ and $\operatorname{coker} \varphi$ are also semistable. Moreover, a Jordan-Hölder filtration on $F, F^{\prime}$ induces a Jordan-Hölder filtration on $\operatorname{ker} \varphi$ and coker $\varphi$, in such a way that the associated graded sheaf of a JordanHölder filtration on $\operatorname{ker} \varphi, \operatorname{coker} \varphi$ is a direct sum component of the associated graded sheaf of a Jordan-Hölder filtration on $F, F^{\prime}$. This immediately implies that the conditions (i)-(iii) of Definition 10.2 hold for $\operatorname{ker} \varphi$ and coker $\varphi$. The condition (iv) holds for $\operatorname{ker} \varphi$ and $\operatorname{coker} \varphi$ tautologically.

Claim 10.4: Suppose that $\operatorname{dim}_{\mathbb{C}} M=2$. Let $F \in \mathcal{C}^{r}$ be a sheaf satisfying the conditions (i)-(iii) of Definition 10.2. Suppose that a Jordan-Hölder filtration on $F$ has length 2 , and let $B_{1}, B_{2}$ be the corresponding hyperholomorphic vector bundles. We have an exact sequence of vector bundles on $\operatorname{Tw}(M \backslash A)$

$$
\left.\left.\left.0 \longrightarrow \operatorname{Tw}\left(B_{1}\right)\right|_{\operatorname{Tw}(M \backslash A)} \longrightarrow F\right|_{\operatorname{Tw}(M \backslash A)} \longrightarrow \operatorname{Tw}\left(B_{2}\right)\right|_{\operatorname{Tw}(M \backslash A)} \longrightarrow 0 .
$$

Let

$$
\nu \in \operatorname{Ext}^{1}\left(\left.\operatorname{Tw}\left(B_{1}\right)\right|_{\operatorname{Tw}(M \backslash A)},\left.\operatorname{Tw}\left(B_{2}\right)\right|_{\operatorname{Tw}(M \backslash A)}\right) \cong H^{1}\left(\operatorname{Tw}\left(\left(B_{1}\right)^{*} \otimes B_{2}\right)\right)
$$

be the corresponding element of the extension group. Denote by $B$ the bundle $\left(B_{1}\right)^{*} \otimes B_{2}$. Consider the image of $\nu$ inside the group

$$
\begin{gathered}
\operatorname{ker}\left(\bigoplus_{x_{i} \in A} \operatorname{Hom}_{\mathbb{C}}\left(\hat{\mathcal{O}}_{s_{x_{i}}}, \mathbb{C}\right) \otimes_{\mathcal{O}_{\mathrm{Tw}(M)}} \operatorname{Tw}(B) \otimes_{\mathcal{O}\left(\mathbb{C} P^{1}\right)} \mathcal{O}(2)\right. \\
\left.\longrightarrow R^{2} \pi_{*} \operatorname{Tw}(B)\right)
\end{gathered}
$$

induced by the exact sequence (7.12). Then (iv) holds if and only if $\nu$ is compatible with the trivialization constructed in (7.16).

Proof: Let $x \in A$ and let $U$ be a small neighbourhood of

$$
s_{x} \backslash I \subset \operatorname{Tw}(M) \backslash(M, I) .
$$


Assume that the fibers of the natural projection $\pi: U \longrightarrow \mathbb{C} P^{1}$ are Stein. Consider the manifold $U^{0}:=U \backslash s_{x}$. The bundle $\left.F\right|_{U_{0}}$ is an extension of $\left.B_{1}\right|_{U^{0}}$ and $\left.B_{2}\right|_{U^{0}}$. This extension might be non-trivial; its non-triviality is controlled by the group

$$
P:=\operatorname{ker}\left(\operatorname{Hom}_{\mathbb{C}}\left(\hat{\mathcal{O}}_{s_{x}}, \mathbb{C}\right) \otimes_{\mathcal{O}_{\operatorname{Tw}(M)}} \operatorname{Tw}(B) \otimes_{\mathcal{O}\left(\mathbb{C} P^{1}\right)} \mathcal{O}(2) \longrightarrow R^{2} \pi_{*} \operatorname{Tw}(B)\right) .
$$

For any $\nu \in P$, and any $J \in s_{x} \backslash I$, consider the restriction $F^{J}$ of $F$ to $\pi^{-1}(J) \subset$ $U^{0}$. Then $\left.F^{J}\right|_{U^{0}}$ is an extension of $\left.B_{1}^{J}\right|_{U^{0}}$ and $\left.B_{2}^{J}\right|_{U^{0}}$. Since $\pi^{-1}(J)$ is a 2dimensional Stein manifold with a point deleted, this extension might be nontrivial; it is controlled by the restriction of $\nu$ to $J$, which is a vector in

$$
\operatorname{ker}\left(\operatorname{Hom}_{\mathbb{C}}\left(\hat{\mathcal{O}}_{x}, \mathbb{C}\right) \otimes_{\mathcal{O}_{(M, J)}} B^{J} \otimes K_{(M, J)} \longrightarrow H^{2}\left(B^{J}\right)\right)
$$

(see Proposition 7.5). The trivialization preserves $F$ and the subsheaf $F_{1} \subset$ $F$, hence it preserves the cohomology class of the extension $\nu$. Therefore, $\nu$ is trivialized. The converse is also clear, because $\left.F\right|_{U}$ is a pushforward of $\left.F\right|_{U^{0}}$ (Lemma 5.5).

Remark 10.5: If $A$ is empty, then for all $F \in \mathcal{C}_{I}^{\text {tw }}$, the sheaf $F$ and all the sheaves $F_{i}$ and $F_{i} / F_{j}$ of Definition 10.2 are non-singular. In this case, $F$ admits a filtration by holomorphic bundles $F_{i}$ such that $F_{i} / F_{i-1}$ is isomorphic to $\operatorname{Tw}\left(B_{i}\right)$, where $\left.B_{i} \cong\left(F_{i} / F_{i-1}\right)\right|_{(M, I)}$, and $\left.F\right|_{(M, I)} \cong \oplus B_{i}$. Moreover, every bundle $F$ on $\operatorname{Tw}(M)$ admitting such a filtration belongs to $\mathcal{C}_{I}^{t w}$. If $M$ is a torus, $\operatorname{dim}_{\mathbb{C}} M>2$, then $A$ is always empty, because all reflexive sheaves on $\operatorname{Tw}(M)$ are locally free, and the associated graded sheaves of the Jordan-Holger filtration are also locally free (this result can be proven in the same fashion as one proves Lemma 8.2 and Proposition 8.4).

Theorem 10.1 is immediately implied by the following theorem.

Theorem 10.6: Let $M$ be a compact hyperkähler torus or a K3 surface, $I$ an induced complex structure, and $L \neq I$ a generic induced complex structure. Assume that $M$ is generic in the sense of having no non-trivial $S U(2)$-invariant integer cycles:

$$
\left(H^{p}(M)_{S U(2)-i n v}\right) \cap H^{p}(M, \mathbb{Z})=0, \quad \text { for all } \quad 0<p<\operatorname{dim}_{\mathbb{R}} M .
$$


Consider the category $\mathcal{C}_{I}^{t w}$ defined above, and let

$$
R_{L}: \mathcal{C}_{I}^{t w} \longrightarrow \mathcal{C}^{r}(M, L)
$$

be the restriction map from $\mathcal{C}_{I}^{t w}$ to the category $\mathcal{C}^{r}(M, L)$ of reflexive sheaves on $(M, I)$. Then (10.1) is an equivalence of categories.

Theorem 10.6 is proven in Subsection 10.3 for $M$ a surface, and in Subsection 10.2 for $M$ a compact torus of dimension greater than 2 .

The definition of $\mathcal{C}_{I}^{t w}$ is motivated by the following heuristic consideration. We need to have a subcategory of the category $\mathcal{C}^{r}$ of reflexive sheaves on $\operatorname{Tw}(M)$ which is isomorphic to $\mathcal{C}^{r}(M, L)$. The category of semisimple objects of $\mathcal{C}^{r}(M, L)$ is embedded to the category of stable bundles on $\operatorname{Tw}(M)$ in a natural way via the twistor transform (see Subsection 6.2). By Proposition 6.3, for any pair of hyperholomorphic bundles $B, B^{\prime}$ on $(M, L)$, we have

$$
\operatorname{Ext}^{1}\left(\operatorname{Tw}(B), \operatorname{Tw}\left(B^{\prime}\right)\right)=\mathbb{C}^{2} \otimes_{\mathbb{C}} \operatorname{Ext}^{1}\left(B, B^{\prime}\right) .
$$

To obtain within $\mathcal{C}^{r}$ a subcategory isomorphic to $\mathcal{C}^{r}(M, L)$, we need to "kill off" half of the extensions. This is performed quite easily by requiring that any extension from $\mathcal{C}_{I}^{t w}$ splits over $(M, I)$. Indeed, by Proposition 6.3, the extensions within the category of coherent sheaves on $\operatorname{Tw}(M)$ are just sections of $\mathcal{O}(1) \otimes$ $\operatorname{Ext}^{1}\left(B, B^{\prime}\right)$. The sections of $\mathcal{O}(1) \otimes \operatorname{Ext}^{1}\left(B, B^{\prime}\right)$ which vanish at $I$ are in 1-to-1 correspondence with $\operatorname{Ext}^{1}\left(B, B^{\prime}\right)$.

If we deal with vector bundles, the definition of $\mathcal{C}_{I}^{t w}$ can be reformulated in a more concise fashion, by saying that $F$ belongs to $\mathcal{C}_{I}^{t w}$ if $F$ is an extension of bundles of type $\operatorname{Tw}\left(B_{i}\right)$ which splits at $(M, I)$.

The rest of the definition of $\mathcal{C}_{I}^{t w}$ adapts the same type of reasoning by accomodating singularities into the picture.

10.2. Reflexive sheaves on generic compact torus and the category $\mathcal{C}_{I}^{t w}$. Let $T$ be a hyperkähler compact torus, $\operatorname{dim}_{\mathbb{C}} T>2$. Assume that all $S U(2)$ invariant integer classes $\eta \in H_{S U(2)-i n v}^{p}(T) \cap H^{p}(T, \mathbb{Z}), 0<p<\operatorname{dim}_{\mathbb{R}} T$ vanish. We are going to prove Theorem 10.6 for the manifold $T$.

All reflexive coherent sheaves on $T$ are bundles (Theorem 8.1). The same argument applied to the twistor space shows that all $F \in \mathcal{C}_{I}^{t w}$ are also bundles. By Proposition 8.4, all bundles on $T$ admit a filtration by vector bundles, with 
associate graded sheaves polystable and locally free. The same argument implies that all $E \in \mathcal{C}_{I}^{t w}$ admit a holomorphic filtration

$$
0=E_{0} \subset E_{1} \subset \ldots \subset E_{n}=E,
$$

with $E_{i} / E_{i-1}=\operatorname{Tw}\left(B_{i}\right)$, where $B_{i}$ is a hyperholomorphic bundle on $(T, I)$, such that $\left.E\right|_{(T, I)} \cong \oplus B_{i}$. Conversely, every such $E$ belongs to $\mathcal{C}_{I}^{t w}$.

Let $L$ be a generic induced complex structure on $T$. Denote by $\mathcal{C}^{b}(T, L)$ the category of holomorphic vector bundles on $(T, L)$. By Theorem 8.1, all reflexive sheaves on $(T, L)$ are vector bundles. Clearly, the category $\mathcal{C}^{b}(T, L)=\mathcal{C}^{r}(T, L)$ is abelian. Consider the restriction functor

$$
R_{L}: \mathcal{C}_{I}^{t w} \longrightarrow \mathcal{C}^{b}(T, L) .
$$

To prove Theorem 10.6 for $T$, we need to show that (10.2) is an equivalence.

As we have indicated above, all simple objects of $\mathcal{C}_{I}^{t w}$ are of form $\operatorname{Tw}(B)$, where $B$ is a stable bundle on $T$. The simple objects of $\mathcal{C}^{b}(T, L)$ are stable bundles as well. Therefore, (10.2) induces an isomorphism of respective subcategories of semisimple objects. We arrive in the situation similar to that described in Corollary 9.7. To apply Corollary 9.7, we need to compare the Yoneda extensions between the semisimple objects in $\mathcal{C}_{I}^{\text {tw }}$ and $\mathcal{C}^{b}(T, L)$.

Let $F, F^{\prime}$ be bundles on $\operatorname{Tw}(T), F=\operatorname{Tw}(B), F^{\prime}=\operatorname{Tw}\left(B^{\prime}\right)$, with $B, B^{\prime}$ hyperholomorphic bundles on $T$. Denote by $B_{L}, B_{L}^{\prime}$ the bundles $B$ and $B^{\prime}$ considered as holomorphic vector bundles on $(T, L)$ :

$$
B_{L}=R_{L}(F), \quad B_{L}^{\prime}=R_{L}\left(F^{\prime}\right) .
$$

By Proposition 6.3, we have

$$
\operatorname{Ext}^{1}\left(F, F^{\prime}\right)=\operatorname{Ext}^{1}\left(B_{L}, B_{L}^{\prime}\right) \otimes_{\mathbb{C}} \mathcal{H}^{0}\left(\mathbb{C} P^{1}, \mathcal{O}(1)\right) .
$$

An extension

$$
0 \longrightarrow F^{\prime} \longrightarrow F^{\prime \prime} \longrightarrow F \longrightarrow 0
$$

belongs to $\mathcal{C}_{I}^{t w}$ if and only if it splits on $(T, I) \subset \operatorname{Tw}(T)$. This allows us to compute the group $\operatorname{Ext}_{\mathcal{C}_{I}^{t w}}^{1}\left(F, F^{\prime}\right)$ of Yoneda extensions in $\mathcal{C}_{I}^{t w}$ from $F$ to $F^{\prime}$. Looking at (10.3), we obtain that

$$
\operatorname{Ext}_{\mathcal{C}_{I}^{t w}}^{1}\left(F, F^{\prime}\right)=\operatorname{Ext}^{1}\left(B_{L}, B_{L}^{\prime}\right) \otimes_{\mathbb{C}} \mathcal{H}^{0}\left(\mathbb{C} P^{1}, \mathcal{O}(1)\right)_{I}
$$


where $\mathcal{H}^{0}\left(\mathbb{C} P^{1}, \mathcal{O}(1)\right)_{I}$ denotes the space of all sections of $\mathcal{O}(1)$ vanishing at $I$. However, the group $\mathcal{H}^{0}\left(\mathbb{C} P^{1}, \mathcal{O}(1)\right)_{I}$ is clearly 1-dimensional, and this gives

$$
\operatorname{Ext}_{\mathcal{C}_{I}^{t w}}^{1}\left(F, F^{\prime}\right) \cong \operatorname{Ext}^{1}\left(B_{L}, B_{L}^{\prime}\right)
$$

The functor

$$
R_{L}: \mathcal{C}_{I}^{t w} \longrightarrow \mathcal{C}^{b}(T, L)
$$

evaluates a section $\eta \in \mathcal{H}^{0}\left(\mathbb{C} P^{1}, \mathcal{O}(1)\right)_{I}$ at $L \in \mathbb{C} P^{1}$, hence it induces an isomorphism on the first Yoneda extensions $\operatorname{Ext}^{1}$, assuming that $L \neq I$. Applying Corollary 9.7, we obtain that

$$
R_{L}: \mathcal{C}_{I}^{t w} \longrightarrow \mathcal{C}^{b}(T, L)
$$

is full and faithful.

To finish the proof of Theorem 10.6 for $T$, it remains to show that $R_{L}$ is surjective on the set of equivalence classes of objects. Let $B$ be a holomorphic bundle on $(T, L)$. We need to show that there is a bundle $F \in \mathcal{C}_{I}^{t w}$ such that $\left.B \cong F\right|_{(M, L)}$. Using Theorem 8.5, we write

$$
B=\left(B_{g r}, \bar{\partial}_{g r}+\nu\right)
$$

where $B_{g r}$ is a flat Hermitian bundle on $T, \bar{\partial}_{g r}$ the holomorphic structure operator on $B_{g r}$ and $\nu \in \Lambda^{0,1}\left(T, \operatorname{End}\left(B_{g r}\right)\right)$ the Higgs field. Denote by $\nabla_{g r}$ the flat Hermitian connection given on $B_{g r}$. Consider the flat connection

$$
\nabla_{\nu}:=\nabla_{g r}+\nu
$$

on $B$ (Corollary 8.7). Lifting $\left(B, \nabla_{\nu}\right)$ as in Theorem 6.2, we obtain a holomorphic bundle $F_{\nu}$ on $\operatorname{Tw}(T)$. Clearly, $F_{\nu} \in \mathcal{C}_{-L}^{t w}$, and $\left.F_{\nu}\right|_{(M, L)}=B$. This proves Theorem 10.6 for $M=T$ and $L=-I$. To prove Theorem 10.6 for arbitrary $L$, we write another flat connection on $B$, as follows.

Let $\alpha \in \mathbb{C}$ be an arbitrary number. Consider the 1 -form $\nu_{\alpha}:=\nu+\alpha \bar{\nu}$.

Claim 10.7: In the above assumptions, the form $\nu_{\alpha}=\nu+\alpha \bar{\nu}$ satisfies the Maurer-Cartan equation:

$$
\nabla_{g r}\left(\nu_{\alpha}\right)+2 \nu_{\alpha} \wedge \nu_{\alpha}=0
$$


Proof: Clearly, $\nu_{\alpha}$ is parallel with respect to the connection $\nabla_{g r}$. To show that $\nu_{\alpha} \wedge \nu_{\alpha}=0$, we need only to prove that $\nu \wedge \bar{\nu}+\bar{\nu} \wedge \nu=0$. However,

$$
\begin{aligned}
(\nu+\bar{\nu}) \wedge(\nu+\bar{\nu}) & =\nu \wedge \nu+\bar{\nu} \wedge \bar{\nu}+\nu \wedge \bar{\nu}+\bar{\nu} \wedge \nu \\
& =\nu \wedge \bar{\nu}+\bar{\nu} \wedge \nu,
\end{aligned}
$$

hence to show $\nu_{\alpha} \wedge \nu_{\alpha}=0$ we need only to prove that $(\nu+\bar{\nu}) \wedge(\nu+\bar{\nu})=0$. Write the connection $\nabla_{r}:=\nabla_{g r}+\nu+\bar{\nu}$. This connection is clearly compatible with the Hermitian structure on $B_{g r}$. The $(0,1)$-part of $\nabla_{r}$ is equal to $\bar{\partial}_{g r}+\nu$, hence its square vanishes, and $\nabla_{r}$ is a holomorphic Hermitian connection. The curvature of $\nabla_{r}$ is written as

$$
\begin{aligned}
\nabla_{r}^{2}=\nabla_{r}(\nu+\bar{\nu})+(\nu+\bar{\nu}) \wedge(\nu+\bar{\nu}) & =\nu \wedge \nu+\bar{\nu} \wedge \bar{\nu}+\nu \wedge \bar{\nu}+\bar{\nu} \wedge \nu \\
& =\nu \wedge \bar{\nu}+\bar{\nu} \wedge \nu
\end{aligned}
$$

because $\nu+\bar{\nu}$ is parallel. Therefore, to prove $\nu \wedge \bar{\nu}+\bar{\nu} \wedge \nu=0$ it means to prove that $\nabla_{r}$ is flat. However, the form $\nu \wedge \bar{\nu}+\bar{\nu} \wedge \nu$ is parallel, hence harmonic. By Remark 3.6, the connection $\nabla_{r}$ is Yang-Mills. Since the Chern classes $c_{1}\left(B_{g r}\right)$, $c_{2}\left(B_{g r}\right)$ vanish, any Yang-Mills connection on $B$ is flat ([Lü], [S]). Therefore, $\nabla_{r}^{2}=0$, and we have $\nu \wedge \bar{\nu}+\bar{\nu} \wedge \nu=0$. We proved Claim 10.7.

By Claim 10.7, the connection $\nabla_{\alpha}:=\nabla_{g r}+\nu+\alpha \bar{\nu}$ is flat. Consider the corresponding holomorphic bundle $F_{\alpha}$ on $\operatorname{Tw}(T)$. Clearly, the $(0,1)$-part of $\nabla_{\alpha}$, taken with respect to $L$, is equal to $\bar{\partial}_{g r}+\nu$. Therefore, $\left.F_{\alpha}\right|_{(T, L)} \cong B$, for all $\alpha$. To prove Theorem 10.6, it remains to show that $F_{\alpha} \in \mathcal{C}_{I}^{t w}$ for some $\alpha$. Let $(\cdot)_{I}^{0,1}$ denote the operation of taking the $(0,1)$-Hodge component with respect to I. Clearly,

$$
F_{\alpha} \in \mathcal{C}_{I}^{t w} \Longleftrightarrow\left(\nu_{\alpha}\right)_{I}^{0,1}=0 .
$$

An elementary calculation insures that if $I \neq \pm L$, then

$$
x(\nu)_{I}^{0,1}=y(\bar{\nu})_{I}^{0,1},
$$

where $x, y$ are non-zero complex numbers, depending on $I, L$. Choosing $\alpha:=-\frac{x}{y}$, we obtain that $\left(\nu_{\alpha}\right)_{I}^{0,1}=0$. By (10.4), this means that $F_{\alpha} \in \mathcal{C}_{I}^{t w}$. We have shown that the full and faithful functor

$$
R_{L}: \mathcal{C}_{I}^{t w} \longrightarrow \mathcal{C}^{b}(T, L)
$$


is surjective on the set of isomorphism classes of objects. Therefore, $R_{L}$ is an equivalence. We proved Theorem 10.6 for $M$ a generic torus of complex dimension $>2$.

10.3. Reflexive sheaves on hyperkähler surfaces. In this Subsection, we prove Theorem 10.6 for $M$ a 2-dimensional torus or a K3 surface. Fix a hyperkähler structure on $M$, and let $L$ be a generic induced complex structure. We have identified the category $C^{r}(M, L)$ with the direct limit

$$
\underset{\lim }{\longrightarrow} \mathcal{C}\left(M \backslash S_{i}, L\right) .
$$

where $S_{i}$ is a sistem of finite subsets of $M$ ordered by inclusion, and $\mathcal{C}\left(M \backslash S_{i}\right)$ the category of coherent sheaves on $M \backslash S_{i}$ which can be extended to coherent sheaves on $M$. If $S$ is non-empty, then the category of coherent sheaves on $\left(M \backslash S_{i}, L\right)$ has cohomological dimension $\leqslant 1$ (Corollary 7.6). Therefore, $C^{r}(M, L)$ has cohomological dimension $\leqslant 1$ as well. Simple objects of this category are stable bundles on $(M, L)$. The simple objects of $\mathcal{C}_{I}^{t w}$ are bundles of the form $\operatorname{Tw}(B)$ on $\operatorname{Tw}(M)$; therefore, the simple objects of $\mathcal{C}_{I}^{t w}$ in 1-to-1 correspondence with the simple (that is, stable) reflexive sheaves on $(M, L)$.

Consider the category

$$
\mathcal{C}^{r}:=\lim _{\longrightarrow} \mathcal{C}^{b}(\operatorname{Tw}(M \backslash S))
$$

(see Subsection 10.1). Let $\mathcal{C}^{t w} \subset \mathcal{C}^{r}$ be a full subcategory of $\mathcal{C}^{r}$ consisting of all extensions of coherent sheaves which have locally free reflexizations of form $\operatorname{Tw}(B)$, where $B$ is a hyperholomorphic bundle. Clearly, $\mathcal{C}_{I}^{t w}$ is a full subcategory of $\mathcal{C}^{t w}$. By Proposition 7.9, we have

$$
\operatorname{Ext}_{\mathcal{C}^{r}}^{2}\left(\operatorname{Tw}(B), \operatorname{Tw}\left(B^{\prime}\right)\right)=0
$$

for all hyperholomorphic bundles $B, B^{\prime}$. Therefore, in $\mathcal{C}^{t w}$, the second Yoneda extension between simple objects vanishes. By Lemma $9.4, \mathcal{C}^{\text {tw }}$ has cohomoloical dimension $\leqslant 1$. Now Lemma 9.5 implies that $\mathcal{C}_{I}^{t w}$ has cohomological dimension $\leqslant 1$ as well.

We arrive to assumptions of Proposition 9.6: a functor of abelian categories of finite length and cohomological dimension $\leqslant 1$ induces an isomorphism on the respective categories of semisimple objects. 
To prove Theorem 10.6 it remains to show that $R_{L}: \mathcal{C}_{I}^{t w} \longrightarrow \mathcal{C}^{r}(M, L)$ induces an isomorphism on the first Yoneda extensions between simple objects.

Let $\nu$ be an Yoneda extension from $\mathcal{C}_{I}^{t w}$,

$$
\nu \in \operatorname{Ext}_{\mathcal{C}_{I}^{t w}}^{1}\left(F_{1}, F_{2}\right),
$$

where $F_{1}, F_{2} \in \mathcal{C}_{I}^{t w}$ are semisimple objects. Then $F_{i}=\operatorname{Tw}\left(B_{i}\right)$, where $B_{i}$ is a hyperholomorphic bundle on $M$. Furthermore, $\nu$ is an element of

$$
H^{0}\left(R^{1} \pi_{*}\left(\left.F_{1}^{*} \otimes_{\mathcal{O}_{\mathrm{Tw}(M)}} F_{2}\right|_{\operatorname{Tw}(M \backslash S)}\right)\right)
$$

where $S$ is a finite set. The restriction of $\nu$ to $(M \backslash S, I) \subset \operatorname{Tw}(M \backslash S)$ vanishes, and the image $r(\nu)$ of $\nu$ inside

$$
\begin{gathered}
P:=\operatorname{ker}\left(\oplus_{x_{i} \in S} \operatorname{Hom}_{\mathbb{C}}\left(\hat{\mathcal{O}}_{s_{x_{i}}}, \mathbb{C}\right) \otimes_{\mathcal{O}_{\mathrm{Tw}(M)}} \operatorname{Tw}\left(B_{1}^{*} \otimes B_{2}\right) \otimes_{\mathcal{O}\left(\mathbb{C} P^{1}\right)} \mathcal{O}(2)\right. \\
\left.\longrightarrow R^{2} \pi_{*} \operatorname{Tw}\left(B_{1}^{*} \otimes B_{2}\right)\right)
\end{gathered}
$$

is trivialized over $\mathbb{C} P^{1} \backslash I$ as in (7.16) (see Claim 10.4).

By Proposition 7.9, we have an exact sequence

$$
\begin{aligned}
0 \longrightarrow & \operatorname{Ext}^{1}\left(B_{1}, B_{2}\right) \otimes H_{\mathbb{C} P^{1}}^{0}(\mathcal{O}(1)) \longrightarrow \operatorname{Ext}^{1}\left(\left.F_{1}\right|_{\mathrm{Tw}(M \backslash S)},\left.F_{2}\right|_{\mathrm{Tw}(M \backslash S)}\right) \\
& \stackrel{r}{\longrightarrow} P \longrightarrow 0 .
\end{aligned}
$$

An extension of reflexive sheaves

$$
\nu \in \operatorname{Ext}^{1}\left(\left.F_{1}\right|_{\operatorname{Tw}(M \backslash S)},\left.F_{2}\right|_{T \mathrm{w}(M \backslash S)}\right)
$$

belongs to $\mathcal{C}_{I}^{t w}$ iff $r(\nu)$ is trivialized over $\mathbb{C} P^{1} \backslash I$ and $\left.\nu\right|_{(M, I)}=0$. Since $r(\nu)$ is trivialized over $\mathbb{C} P^{1} \backslash I$, the restriction $\left.r(\nu)\right|_{\mathbb{C P}^{1} \backslash I}$ is determined by its value at any point $L \in \mathbb{C} P^{1}, L \neq I$. Similarly, any section of

$$
\operatorname{Ext}^{1}\left(B_{1}, B_{2}\right) \otimes H_{\mathbb{C} P^{1}}^{0}(\mathcal{O}(1))
$$

vanishing at $I$ is determined by its restriction on $(M, L)$. Therefore, $R_{L}$ : $\mathcal{C}_{I}^{t w} \longrightarrow \mathcal{C}^{r}(M, L)$ induces an embedding on $\operatorname{Ext}^{1}$ between semisimple objects. To show that this map is an isomorphism, take

$$
\alpha \in \operatorname{Ext}_{\mathcal{C}^{b}(M \backslash S, L)}^{1}\left(B_{1}, B_{2}\right),
$$


where $B_{1}, B_{2}$ are hyperholomorphic bundles considered as holomorphic bundles over $(M, L)$. By Proposition 7.2 , any trivialized over $\mathbb{C} P^{1} \backslash I$ section of $\left.P\right|_{\mathbb{C} P^{1} \backslash I}$ can be extended to $\mathbb{C} P^{1}$. Therefore, the image

$$
\begin{aligned}
r(\alpha) \in P_{L} & :=\operatorname{ker}\left(\operatorname{Hom}_{\mathbb{C}}\left(\hat{\mathcal{O}}_{(M, L), x}, \mathbb{C}\right) \otimes_{\mathcal{O}_{(M, L)}} K(M, L) \otimes B_{1}^{*} \otimes B_{2}\right. \\
& \left.\longrightarrow H^{2}\left((M, L), B_{1}^{*} \otimes B_{2}\right)\right)
\end{aligned}
$$

can be extended to a section of $P$, on the whole $\mathbb{C} P^{1}$. Clearly, any element in $\operatorname{Ext}^{1}\left(B_{1}, B_{2}\right)$ can be extended to a unique section of $\operatorname{Ext}^{1}\left(B_{1}, B_{2}\right) \otimes H_{\mathbb{C} P^{1}}^{0}(\mathcal{O}(1))$ vanishing at $I$. We have a commutative diagram of long exact sequences, with the rightmost and the leftmost vertical arrows isomorphisms

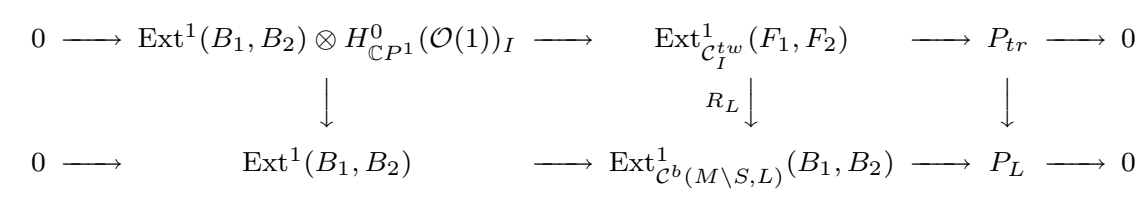

where $H_{\mathbb{C} P^{1}}^{0}(\mathcal{O}(1))_{I}$ denotes the space of sections of $\mathcal{O}(1)$ vanishing at $I, P_{t r}$ denotes the space of sections of $P$ which are trivialized over $\mathbb{C} P^{1} \backslash I$, and the vertical maps are restrictions to $(M, L) \subset \mathrm{Tw}(M)$.

The middle vertical arrow of (10.7) is also an isomorphism, by the snake lemma; therefore, $R_{L}$ induces an isomorphism on Yoneda extensions of semisimple objects. This proves Theorem 10.6 for $M$ a 2-dimensional torus or a K3 surface. Theorem 10.6 is proven.

\section{Coherent Sheaves With isolated Singularities}

The main result of this Section is the following

Theorem 11.1: Let $M$ be a hyperkähler K3 surface or a compact torus without non-trivial integer $S U(2)$-invariant classes, and $L, L^{\prime}$ generic induced complex structures. Consider the categories $\mathcal{C}(M, L), \mathcal{C}\left(M, L^{\prime}\right)$ of coherent sheaves on $(M, L)$ and $\left(M, L^{\prime}\right)$. Then the categeries $\mathcal{C}(M, L), \mathcal{C}\left(M, L^{\prime}\right)$ are equivalent. Moreover, this equivalence can be chosen canonically if we fix an induced complex structure $I \neq L, L^{\prime}$. 
Proof: Let $F \in \mathcal{C}(M, L)$, and $F^{* *}$ its reflexization, which is a bundle. Fix an induced complex structure $I \neq L, L^{\prime}$. As in the proof of Theorem 10.1, we consider the category $\mathcal{C}_{I}^{t w}$. We have shown that the restriction $R_{L}: \mathcal{C}_{I}^{t w} \longrightarrow \mathcal{C}^{r}(M, L)$ defines an equivalence, where $\mathcal{C}^{r}(M, L)$ is the category of reflexive sheaves on $(M, L)$ (Theorem 10.6). Denote by $E \in \mathcal{C}_{I}^{t w}$ the reflexive sheaf which corresponds to $F^{* *}$ under this equivalence.

Our argument uses the local twistor geometry of a hyperkähler manifold, following Subsection 7.1 (see also [V4], 6.2). The following claim is trivial.

Claim 11.2: In the above assumptions, let $E \in \mathcal{C}_{I}^{t w}$, and let $x$ be any point in $M$. Then the bundle $E$ is canonically trivialized in a neighbourhood of $s_{x} \backslash I$, where $s_{x} \subset \operatorname{Tw}(M)$ is a horisontal twistor section.

Proof: Let $x$ be a point in $A$, where $A \times\{I\}$ is a singular set of $E$ which is used in the definition of $\mathcal{C}_{I}^{t w}$. In a neighbourhood of $s_{x} \backslash I$, the sheaf $E$ is trivialized, by definition of $\mathcal{C}_{I}^{t w}$. If $x \notin A$, then $E$ in a neighbourhood of $s_{x}$ is a successive extension of the bundles of form $\operatorname{Tw}\left(B_{i}\right)$. Each of these bundles is equal to $\mathcal{O}_{\mathbb{C} P}^{\oplus P_{i}}$ on $s_{x}$, and therefore, $\left.E\right|_{s_{x}}$ is also trivial. Using (7.5), we obtain a trivialization of $E$ in a neighbourhood of $s_{x} \backslash I$, for all $x \in M$.

We are going to attach singularities to $E$ in such a way that the equivalence $R_{L}: \mathcal{C}_{I}^{t w} \longrightarrow \mathcal{C}^{b}(M, L)$ can be extended to the sheaves with isolated singularities.

We prove Theorem 11.1 in the same fashion as we proved Theorem 10.1. Just as in Theorem 10.6, we shall construct an intermediate category $\widetilde{\mathcal{C}}_{I}^{t w}$, which is a subcategory of the category of coherent sheaves on the twistor space. We show that the natural restriction functor from $\widetilde{\mathcal{C}}_{I}^{t w}$ to $\mathcal{C}(M, L)$ is an equivalence, if $L$ is a generic complex structure distinct from $I$. Since $L$ can be chosen arbitrarily, this will imply immediately that $\mathcal{C}(M, L)$ is equivalent to $\mathcal{C}\left(M, L^{\prime}\right)$.

Definition 11.3: The category $\widetilde{\mathcal{C}}_{I}^{t w}$ is defined as follows.

An object of $\widetilde{\mathcal{C}}_{I}^{t w}$ is a coherent sheaf $F$ on $\operatorname{Tw}(M)$ satisfying the following conditions.

(i): The reflexization $E:=F^{* *}$ belongs to $\mathcal{C}_{I}^{t w}$, where the category $\mathcal{C}_{I}^{t w}$ is defined as in Definition 10.2.

(ii): The sheaf $F$ is non-singular outside of $A \times \mathbb{C} P^{1} \subset \mathrm{Tw}(M)$, where $A \subset M$ is a finite set. 
(iii): Let $Z:=\{A\} \times I \subset(M, I) \subset \operatorname{Tw}(M)$ be the finite subset of $(M, I) \subset$ $\operatorname{Tw}(M)$ corresponding to $A$, and $j: \operatorname{Tw}(M) \backslash Z \hookrightarrow \operatorname{Tw}(M)$ the natural embedding. Then the canonical homomorphism $F \longrightarrow j_{*} j^{*} F$ is an isomorphism.

(iv): In the notation of of Subsection 7.1, consider the restriction of $F$ and of $E=F^{* *}$ to $\operatorname{Tw}(M)_{x_{0}, I}$. Let $\left.E\right|_{\operatorname{Tw}(M)_{x_{0}, I}} \stackrel{\tau_{E}}{\longrightarrow} \xi^{*} E_{S}$ be the trivialization constructed in Claim 11.2. Then there exists a sheaf $F_{S}$ equipped with a map $F_{S} \stackrel{\rho_{F_{S}}}{\longrightarrow} E_{S}$ which is equivalence outside of $x_{0},{ }^{1}$ and an isomorphism

$$
\left.F\right|_{\operatorname{Tw}(M)_{x_{0}, I}} \stackrel{\tau_{F}}{\longrightarrow} \xi^{*} F_{S}
$$

such that the following diagram is commutative

$$
\begin{array}{cc}
\left.F\right|_{\operatorname{Tw}(M)_{x_{0}, I}} \stackrel{\tau_{F}}{\longrightarrow} \xi^{*} F_{S} \\
\rho_{F} \downarrow \\
\left.E\right|_{\operatorname{Tw}(M)_{x_{0}, I}} \stackrel{\tau^{*}\left(\rho_{F_{S}}\right)}{\longrightarrow} \xi^{*} E_{S}
\end{array}
$$

(Here, $\rho_{F}$ and $\rho_{F_{S}}$ denote the respective reflexization maps). This means that the singularities of $F$ are compatible with the local trivialization of $E$ constructed in Subsection 7.1.

The morphisms of $\widetilde{\mathcal{C}}_{I}^{t w}$ are morphisms of coherent sheaves.

Remark 11.4: Clearly, the sheaf $F_{S}$ and the morphism $F_{S} \longrightarrow E_{S}$ can be reconstructed in a canonical way from $F$. Take for $S$ the neighbourhood of $x_{0}$ in $(M, L)$. Then $E_{S}$ is the restriction of $E$ to $S \subset(M, L) \subset \operatorname{Tw}(M)$, and $\left.F_{S} \cong F\right|_{S}$.

The following theorem implies Theorem 11.1, as we indicated in the beginning of this Section.

Theorem 11.5: Let $M$ be a compact hyperkähler manifold, $I$ an arbitrary induced complex structure, and $\widetilde{\mathcal{C}}_{I}^{t w}$ the category constructed above. Consider a generic induced complex structure $L$, and let

$$
R_{L}: \widetilde{\mathcal{C}}_{I}^{t w} \longrightarrow \mathcal{C}(M, L)
$$

\footnotetext{
${ }^{1}$ This means that $\rho_{F_{S}}$ is the reflexization map.
} 
be the restriction map, $\left.F \longrightarrow F\right|_{(M, L)}$. Then (11.1) is an equivalence of categories.

Proof: We construct the inverse functor $\Upsilon: \mathcal{C}(M, L) \longrightarrow \widetilde{\mathcal{C}}_{I}^{t w}$ as follows. Take $F_{L} \in \mathcal{C}(M, L)$. Let $E_{L}$ be its reflexization, and $E \in \mathcal{C}_{I}^{t w}$ the corresponding sheaf over the twistor space, which is defined in a canonical way by Theorem 10.6.

Let $x_{0} \in M$ be a singular point of $F_{L}$. Consider the infinitesimal neighbourhood of

$$
\left\{x_{0}\right\} \times\left(\mathbb{C} P^{1} \backslash I\right) \subset \mathrm{Tw}(M) \backslash(M, I)
$$

in $\operatorname{Tw}(M) \backslash(M, I)$, denoted, as in Subsection 7.1, by $\operatorname{Tw}(M)_{x_{0}, I}$. Then $E$ is trivialized over $\operatorname{Tw}(M)_{x_{0}, I}$, and we may write $\left.E\right|_{\operatorname{Tw}(M)_{x_{0}, I}}=\xi^{*}\left(E_{L, x_{0}}\right)$, where $E_{L, x_{0}}$ is the germ of $E_{L}$ in $x_{0}$. Let $\left.F\right|_{\operatorname{Tw}(M)_{x_{0}, I}}:=\xi^{*}\left(F_{L, x_{0}}\right)$ be the sheaf corresponding to $F_{L}$. Since $F_{L}$ has isolated singularities, the sheaves $\left.F\right|_{\operatorname{Tw}(M)_{x_{0}, I}}$ and $\left.E\right|_{\operatorname{Tw}(M)_{x_{0}, I}}$ are canonically isomorphic outside of $\left\{x_{0}\right\} \times\left(\mathbb{C} P^{1} \backslash I\right)$. Gluing together $\left.F\right|_{\operatorname{Tw}(M)_{x_{0}, I}}$ and $E$, we obtain a sheaf $F_{0}$ on $\operatorname{Tw}(M) \backslash(M, I)$. Let $A$ be the singular set of $F_{L}$. Then $E$ is equal to $F_{0}$ outside of $\{A\} \times \mathbb{C} P^{1} \subset \operatorname{Tw}(M)$, hence $F_{0}$ can be extended smoothly from $\operatorname{Tw}(M) \backslash(M, I)$ to a sheaf $F_{1}$ on $\operatorname{Tw}(M) \backslash Z$, where $Z=\{A\} \times\{I\}$ is the finite set defined in Definition 11.3. Consider the sheaf $j_{*}\left(F_{1}\right)$, where $j: \operatorname{Tw}(M) \backslash Z \hookrightarrow \operatorname{Tw}(M)$ is the natural embedding.

If $F_{L}$ is torsion-free, then $F_{1}$ is also torsion-free. In this case we set $\Upsilon\left(F_{L}\right):=$ $j_{*}\left(F_{1}\right)$; this sheaf is coherent because $Z$ has codimension $\geqslant 3(Z$ is by construction a finite set).

If $F_{L}$ has torsion, then $j_{*}\left(F_{1}\right)$ is quasicoherent. We realize $\Upsilon\left(F_{L}\right)$ as a subsheaf in $j_{*}\left(F_{1}\right)$, in a canonical way. Outside of $Z$, we set $\Upsilon\left(F_{L}\right)$ to $j_{*}\left(F_{1}\right)$. Let $\gamma \in j_{*}\left(F_{1}\right)$ be a section defined in a neighbourhood of a point $z \in Z$. Then $\gamma$ belongs to $\Upsilon\left(F_{L}\right) \subset j_{*}\left(F_{1}\right)$ if $\gamma$ is generated over $\mathcal{O}_{\mathrm{Tw}(M)}$ by the sections of $F_{1}$ which are compatible with the trivialization

$$
\left.F_{1}\right|_{\mathrm{Tw}(M)_{z, I}}=\xi^{*}\left(F_{L, z}\right)
$$

constructed above. 
Since $F_{L, z}$ is coherent, it is finitely generated over $\mathcal{O}_{(M, I)}$. For every germ of $F_{L, z}$, there exists a unique section of $\left.F_{1}\right|_{\mathrm{Tw}(M)_{z, I}}$ which is compatible with the trivialization (11.2). Therefore, the sheaf $\Upsilon\left(F_{L}\right)$ is finitely generated. This proves that $\Upsilon\left(F_{L}\right)$ is coherent.

By construction, the sheaf $\Upsilon\left(F_{L}\right)$ satisfies the conditions of Definition 11.3. This construction is clearly functorial. Indeed, it is functorial on bundles by Theorem 10.6, and the sheaf $F_{L}$ is just a bundle with some singularities attached; every morphism of sheaves from $\mathcal{C}_{I}$ induces a morphism on the corresponding reflexization bundles, which is compatible with the trivialization (7.5), hence extends naturally to isolated singularities.

We proved Theorem 11.5. Theorem 11.1 is proven.

From Theorem 4.5 and Theorem 11.1, the following result is apparent.

Theorem 11.6: Let $M$ be a compact torus or a K3 surface, and $L, L^{\prime}$ complex structures of Kähler type. Assume that $L$ and $L^{\prime}$ are Mumford-Tate generic. Then the categories $\mathcal{C}(M, L), \mathcal{C}\left(M, L^{\prime}\right)$ of coherent sheaves on $(M, L)$ and $\left(M, L^{\prime}\right)$ are equivalent.

Proof: Consider the sequence $\mathcal{H}_{1}, \mathcal{H}_{2}, \ldots \mathcal{H}_{n}$ of hyperkähler structures, satisfying the assumptions of Theorem 4.5, and let $L_{0}=L, L_{2}, L_{3}, \ldots, L_{n+1}=L^{\prime}$ be the corresponding sequence of Mumford-Tate generic complex structures. By Claim 4.4, the complex structures $L_{k}$ are generic. Applying Theorem 11.1, we find that the following categories are equivalent:

$$
\mathcal{C}\left(M, L_{k}\right) \sim \mathcal{C}\left(M, L_{k+1}\right)
$$

Therefore, $\mathcal{C}\left(M, L_{0}\right)=\mathcal{C}(M, L)$ is equivalent to $\mathcal{C}\left(M, L_{n+1}=\mathcal{C}\left(M, L^{\prime}\right)\right.$. We proved Theorem 11.6.

Acknowledgements: I am grateful to V. Ginzburg, who asked about the categories of coherent sheaves on a K3 surface, and D. Kaledin for many hours of stimulating discussions. Many thanks to I. Dolgachev, D. Kazhdan, A. Tyurin and A. Vaintrob for insightful comments and useful advice. The referee was very helpful in correcting the most insidious errors and omissions. 


\section{REFERENCES}

[BT] Babenko I. K., Taimanov I. A., Massey products in symplectic manifolds, math.SG/9911132 (also in: Sb. Math. 191 (2000), 1107-1146).

[BS] Bando, S., Siu, Y.-T, Stable sheaves and Einstein-Hermitian metrics, In: Geometry and Analysis on Complex Manifolds, Festschrift for Professor S. Kobayashi's 60th Birthday, ed. T. Mabuchi, J. Noguchi, T. Ochiai, World Scientific, 1994, pp. 39-50.

[Bes] Besse, A., Einstein Manifolds, Springer-Verlag, New York (1987)

[Bo] Bogomolov, F. A., The decomposition of Kähler manifolds with a trivial canonical class, Mat. Sb. (N.S.) 93 (135) (1974), 573-575, 630.

[C] Calabi, E., Métriques kähleriennes et fibrès holomorphes, Ann. Ecol. Norm. Sup. 12 (1979), 269-294.

[DGMS] Deligne, P., Griffiths, Ph., Morgan, J., Sullivan, D., Real homotopy theory of Kähler manifolds, Invent. Math. 29 (1975), no. 3, 245-274.

[GM] Goldman, W. M., Millson, J. J. The deformation theory of representations of fundamental groups of compact Kähler manifolds, Inst. Hautes Etudes Sci. Publ. Math. No. 67 (1988), 43-96.

[GH] Griffiths, Ph., Harris, J., Principles of Algebraic Geometry, Wiley-Intersience, New York, 1978.

[GH] Hartshorne, R. Local cohomology, A seminar given by A. Grothendieck, Harvard University, Fall, 1961, LNM 41, Springer-Verlag, 1967.

[HKLR] N.J. Hitchin, A. Karlhede, U. Lindström, M. Roček, Hyperkähler metrics and supersymmetry, Comm. Math. Phys (1987).

[KV] Kaledin, D., Verbitsky, M., Non-Hermitian Yang-Mills connections, alg-geom 9606019 (also in: Selecta Math. (N.S.) 4 (1998), no. 2, 279-320)

[Kob] Kobayashi S., Differential geometry of complex vector bundles, Princeton University Press, 1987.

[Kon] Kontsevich, M., Homological Algebra of Mirror Symmetry, talk at ICM, Zurich, 1994 (alg-geom/9411018).

[LY] Li, Jun, and Yau, S.-T., Hermitian Yang-Mills connections on non-Kahler manifolds, in "Mathematical aspects of string theory" (S.T. Yau ed.), World Scientific Publ., London, 1987, pp. 560-573.

[Lü] M. Lübke, Chernklasses von Hermite-Einstein Vektorbündeln, Math. Ann. 260 (1982), 133-141.

[May] May, P. J. Matric Massey products, J. Algebra 121969 533-568.

[Oo] Oort, F. Yoneda extensions in abelian categories, Math. Ann. 1531964 227-235.

[O] Orlov, D., Equivalences of derived categories and K3 surfaces, alg-geom/9606006 (also in: Algebraic geometry, 7, J. Math. Sci. (New York) 84 (1997), no. 5, 1361-1381).

[OSS] Okonek, C., Schneider, M., Spindler, H., Vector bundles on complex projective spaces. Progress in mathematics, vol. 3, Birkhauser, 1980. 
[Re] Retah, V. S. Massey operations, obstructions to formality in Sullivan's sense and obstructions to Kodaira-Spencer deformations, International Topology Conference (Moscow State Univ., Moscow, 1979). Uspekhi Mat. Nauk 35 (1980), no. 3(213), 217-220.

[Sal] Salamon, S., Quaternionic Kähler manifolds, Inv. Math. 67 (1982), 143-171.

[S] Simpson, C. T. Moduli of representations of the fundamental group of a smooth projective variety, I. Inst. Hautes Etudes Sci. Publ. Math. No. 79 (1994), 47-129.

[ST] Siu, Y. T., Trautmann, G. Deformations of coherent analytic sheaves with compact supports, Mem. Amer. Math. Soc. 29 (1981), no. 238, iii+155 pp.

[T] Tian, G., Gauge theory and calibrated geometry, I, math.DG/0010015 (also in: Annals Math. 151 (2000) 193-268).

[UY] Uhlenbeck K., Yau S. T., On the existence of Hermitian Yang-Mills connections in stable vector bundles, Comm. on Pure and Appl. Math., 39, p. S257-S293 (1986).

[V0] Verbitsky M., Hyperholomorphic bundles over a hyperkähler manifold, alg-geom electronic preprint 9307008 (1993), 43 pages, also published in: Journ. of Alg. Geom., 5 no. 4 (1996) pp. 633-669.

[V1] Verbitsky M., Hyperkähler embeddings and holomorphic symplectic geometry II, alg-geom electronic preprint 9403006 (1994), (also in: GAFA 5 no. 1 (1995), 92-104.)

[V2] Verbitsky, M., Cohomology of compact hyperkähler manifolds and its applications, alggeom electronic preprint 9511009, 12 pages, also published in: GAFA vol. 6 (4) pp. 601-612 (1996).

[V3] Verbitsky M., Hypercomplex Varieties, alg-geom/9703016 (also in: Comm. Anal. Geom. 7 (1999), no. 2, 355-396)

[V4] Verbitsky M., Hyperholomorphic sheaves and new examples of hyperkähler manifolds, alggeom 9712012 - 113 pages, published in a book "Hyperkähler manifolds" (joint with D. Kaledin), International Press, Boston, 2001.

[V5] Verbitsky M., Projective bundles over hyperkähler manifolds and stability of Fourier-Mukai transform, math.AG/0107196 (2001), 60 pages.

[Voi] Voisin, C. A counterexample to the Hodge conjecture for Kähler varieties, math.AG/0112247, 14 pages.

Misha Verbitsky

E-mail: verbit@thelema.dnttm.ru

E-mail: verbit@mccme.ru 